\title{
New approaches to the lithiation kinetics in reaction-limited battery electrodes through electrochemical impedance spectroscopy
}

\author{
Nuria Vicente ${ }^{1}$, Marta Haro ${ }^{2}$, and Germà Garcia-Belmonte ${ }^{1, *}$ \\ 1 Institute of Advanced Materials (INAM), Universitat Jaume I, ES-12006 Castelló, Spain \\ 2 Okinawa Institute of Science and Technology (OIST) Graduate University, 1919-1 \\ Tancha, Onna-son, Okinawa 904-0495, Japan
}

\begin{abstract}
Electrochemical impedance spectroscopy is a widely employed technique probing kinetic limitations in the charging of battery electrodes. Hindrance mechanisms locate at the interfaces between the active material and the electrolyte, and in the bulk of the reacting compound. Rate-limiting mechanisms are viewed as resistive circuit elements and can be extracted by standard impedance analyzers. Classical impedance models consider charge transport, mainly ion diffusion as slower carrier, as the principal kinetic limitation impeding full electrode charging. This is indeed the case for many technologically relevant battery compounds. In other instances, instead of being diffusion-limited, electrodes may undergo charging limitation caused by the kinetics of the reduction reaction itself. Specific impedance models for reaction-limited mechanisms are summarized here and proved for relevant electrode compounds, in particular for conversion or alloying electrodes in which $\mathrm{Li}^{+}$intake produces a full rearrangement of the lattice structure with significant atomic displacement.
\end{abstract}

Keywords: battery electrode, electrochemical impedance spectroscopy, lithiation kinetics, reaction-limited charging 


\section{Content}

Introduction

Background: elementary circuit elements

Basic impedance models

Cathodes: $\mathrm{LiFePO}_{4}$ and $\mathrm{Li}\left[\mathrm{Ni}_{y} \mathrm{Co}_{z} \mathrm{Mn}_{1-y-z}\right] \mathrm{O}_{2}$

Anodes: MWCT@ $\mathrm{TiO}_{2}$ and $\mathrm{MAPbBr} 3$

Conversion and alloying anodes: $\mathrm{FeOOH}, \mathrm{ZnFe}_{2} \mathrm{O}_{4}$ and $\mathrm{Si} / \mathrm{Ge}$

$\mathrm{Li}^{-} \mathrm{O}_{2}$ impedance response

Conclusion 


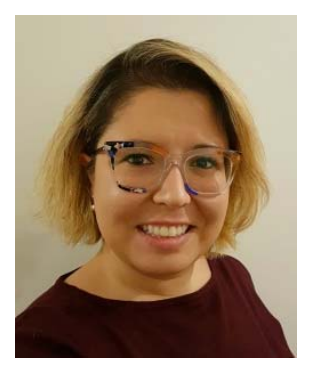

Nuria Vicente is currently a $\mathrm{PhD}$ student in Sciences at Universitat Jaume I (Castelló), Spain. She received a Master's degree in Applied Physics at Universitat Jaume I in 2014. From 2013, she is a member of Institute of Advanced Materials where she develops research activity on new perovskite-based materials for storage energy and analysis of electrode kinetics using electrochemical impedance spectroscopy under the supervision of Prof. Germà GarciaBelmonte.

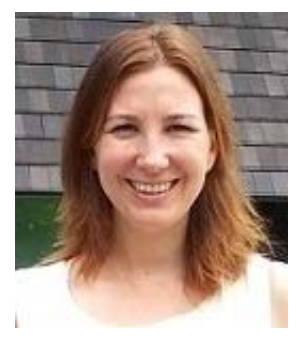

Marta Haro ( $\mathrm{PhD}$ in Physical Chemistry, Universidad de Zaragoza, Spain, 2007) is a staff scientist at Okinawa Institute of Science and Technology (Japan). Prior to joining OIST, she worked as postdoctoral researcher in the Photovoltaic and Optoelectronic Devices group at Universitat Jaume I (Spain), in Adsorption and Environmental Protection on Porous Solids at Instituto Nacional de Carbon (Spain), and in Organophotonics group at University of Hull (UK). Her research interest is focused on the development of electrodes and their electrochemical and photoelectrochemical analysis for energy storage in Li-ion batteries, and other applications such as solar fuel generation from water splitting and photodegradation processes in water treatment. 


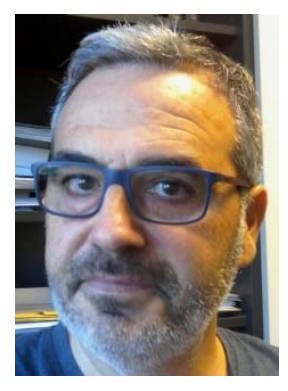

Germà Garcia-Belmonte joined the Universitat Jaume I de Castelló in 1992 and currently works as a Full Professor of Applied Physics (since 2010) at the Institute of Advanced Materials (INAM) which develops research activities on materials, nanostructures and devices for production and efficient use of clean energies. He studied intercalation processes in oxides and polymer films by impedance methods. He also conducted researches in various areas within the field of Organic Electronics and photovoltaics as electronic mechanisms in organic light-emitting diodes, organic photovoltaics, and plastic and thin-film solar cells. He is currently active in the topic of perovskite-based solar cells. Also of interest is the electrochemical kinetics of electrodes for batteries. Device physics using impedance spectroscopy (including modeling and measuring) is his main subject. 


\section{Introduction.}

Electrochemical impedance spectroscopy (EIS) has been widely employed for decades as a standard characterization tool of battery electrodes. It consists on the application of a small perturbation (usually a sinusoidal voltage) that slightly displaces the charge state of the electrode from a given steady state. The perturbation $(10-20 \mathrm{mV})$ explores different time scales, usually in the frequency range of $1 \mathrm{MHz}$ down to $1 \mathrm{mHz}$. This small deviation induces changes in transport and charging mechanisms involving ionic species (mainly Liion in our case) of different time scales, response times or frequencies. As a consequence, EIS allows exploring the charging kinetics in battery electrodes using a non-destructive and easy-to-use experimental technique incorporated in commonly employed electrochemical testing systems. EIS is complementary to other well-established procedures such as the analysis of galvanostatic charge/discharge curves at different current intensities to determine the electrode rate capability and power response. In combination with structural techniques, EIS makes accessible the connection between particular electrochemical mechanisms and electrode morphology and constituents. This is the key point why EIS is a testing tool of absolute necessity in any electrochemical laboratory.

The analysis of EIS spectra $Z(\omega)$, being $\omega$ the angular frequency of the perturbation, is usually accomplished by fitting a proposed equivalent circuit to the data collected by the impedance analyzer. Elaboration of equivalent circuits able to capture the essential electrochemical mechanisms involved in the electrode response needs of theoretical knowledge about capacitive (charging) and resistive (hindrance) processes occurring at the electrode. These processes can be located at the interfaces between the active material and the electrolyte, and in the bulk of the reacting compound. Limitations to electrode charging stem from interface charge transfer mechanisms, as well as inner processes of the host. Rate-limiting mechanisms directly point to resistive circuit elements, which can be straightforwardly identified in the impedance plots in the complex plane $\left(Z^{\prime \prime}-Z^{\prime}\right)$ for data visualization. Therefore, one can expect resistances accounting both for interfacial and material bulk hindrance processes. Classical impedance models highlighted charge transport, mainly ion diffusion as slower carrier, as the principal limitation impeding full electrode charging. It is restriction to ion motion what delays full electrode reduction reaction and limits rate capability. This is so in many technologically relevant battery compounds. However, it cannot be discarded other origins for the delays in full charging. 
Instead of being diffusion-limited, electrodes may undergo charging limitation caused by the kinetics of the reduction reaction itself. In these cases, we can think about reactionoriginated charging limitation. This mechanism should be characterized by a corresponding resistive element similarly to that encountered for diffusion-limited electrodes. Because this is a much less explored process, the kinetics of reaction limitation, not related to the charge transport, will be addressed in this Featured Article.

The work is organized starting from relating resistive and capacitive circuit elements to the known electrochemical processes occurring in the electrode. Elementary circuit elements are then combined to provide full equivalent circuits mostly used for analyzing the kinetics mechanisms in battery compounds. Here, we give a brief summary of diffusion models and introduce the electrical representation for reaction-limited electrode analysis. This is accomplished by comparing the expected impedance spectra for the two cases, diffusionand reaction-limited, signaling similarities and differences. After this brief outline about useful circuit models, the response of several electrode compounds is shown in the light of the impedance modeling. Both cathode and anode electrodes $\left(\mathrm{LiFePO}_{4}, \mathrm{Li}\left[\mathrm{Ni}_{y} \mathrm{Co}_{z} \mathrm{Mn}_{1-y-}\right.\right.$ z] $\mathrm{O}_{2}$, and $\mathrm{TiO}_{2}$ ) are addressed indicating the potential use of the reaction models in a phenomenological way. These models are fully justified in the case of conversion and alloying electrodes for which a complete rearrangement of the material structure is expected. Here, the analysis is focused on several electrode materials with different morphologies $\left(\mathrm{FeOOH}, \mathrm{ZnFe}_{2} \mathrm{O}_{4}\right.$ and $\left.\mathrm{Si} / \mathrm{Ge}\right)$. To spread out the use of the impedance technique, we also present recent results and equivalent circuit interpretation of the impedance response of $\mathrm{Li}_{2} \mathrm{O}_{2}$ electrodes. The Feature Article makes up an accessible summary to the application of electrochemical impedance spectroscopy to the study of the kinetics of battery electrode charging, stressing those cases limited by reaction-originated mechanisms.

\section{Background: elementary circuit elements.}

Before summarizing the main impedance models and most commonly used equivalent circuits of application in the battery analysis, it is interesting pointing out some general features of the elementary circuit elements. Detailed information and theoretical background can be easily found in books for specialists, ${ }^{1}$ so our approach will be merely descriptive. Because battery electrodes are immersed in electrolytes containing a lithium reservoir, impedance measurement entails perturbation of processes at the 
electrode/electrolyte interface, in addition to those occurring within the electrode bulk material. When impedance spectra are analyzed, mechanisms at the outer interfaces (electrode/electrolyte) usually involve both capacitive and resistive processes. One of them is the ability of the interface to accumulate ionic species in the vicinity of the surface. The applied voltage modulates the amount of ions so as to produce a net capacitance. This is classically known as the double-layer capacitance $C_{\mathrm{dl}}$ because the interface charging occurs similarly to an electrical capacitor. Ideally, the double-layer capacitance, which may respond to a complex ionic distribution comprising Helmholtz and diffuse layers and the specific polarization features, is a function of the dielectric properties of the interface. If an effective permittivity is defined for the contacting materials near the interface, the doublelayer capacitance (per unit interface area) can be simply expressed as

$$
C_{\mathrm{dl}}=\frac{\varepsilon \varepsilon_{0}}{d}
$$

Here $\varepsilon$ accounts for the effective dielectric constant, $\varepsilon_{0}$ is the vacuum permittivity, and $d$ represents the effective charge separation. Ions pile up at the interface producing thin space charge layers with net separation in the range of 1-10 $\AA$. Using equation 1, these distances imply double-layer capacitance values of the order of $C_{\mathrm{dl}} \approx 10-100 \mu \mathrm{F} \mathrm{cm}^{-2}$, depending on the permittivity. The obvious strategy to increase the value of the total double-layer capacitor is enlarging the interface area by means of porous matrices that allow the electrolyte penetration and the formation on an extended double layer. For high porosities, $C_{\mathrm{dl}}$ can attain values orders of magnitude in excess of the capacitive level shown by planar electrodes. The final value obtained for $C_{\mathrm{dl}}$ is indeed an indication of the porous degree of the electrode, becoming a useful guide for proper impedance model selection, as later explained.

There is a process related to the formation of the ionic double layer that involves the interchange of charge carrier between the two constituents of the interface. This is usually labeled as a charge transfer mechanism that entails the injection/extraction of electronic carriers to/from the electrode material or the insertion/release of ionic species. In any case, the ionic (or electronic) charge transfer process occurs by surpassing an interfacial potential barrier. It is then governed by the energetic properties of the interface at a given charging state. Any barrier crossing involving charge carriers is accompanied by an energy loss, which in electrical terms is always modeled by means of resistive elements. This is why $C_{\mathrm{dl}}$ parallels a current-related circuit element usually known as charge transfer resistance $R_{\mathrm{ct}}$. It is then a measurement of the interface permeability to the pass of charge. 
While $C_{\mathrm{dl}}$ usually exhibits rather voltage-independent responses, as expected for dielectric capacitances, $R_{\mathrm{ct}}$ exhibits a dependence on the voltage related to the charge state of the electrode, usually with smaller values for lithiated electrodes. In some specific conditions $R_{\mathrm{ct}}$ is indeed the rate-limiting process, particularly when the electrode is highly charged and the inner ionic movement is fast enough. In many other cases, its contribution is comparable to the charging hindrance produced by solid state diffusion/reaction mechanisms inside the electrode material. The combination of $C_{\mathrm{dl}}$ and $R_{\mathrm{ct}}$ corresponds to the characteristic frequency commonly observed in impedance spectra as $\omega_{\mathrm{dl}}=1 / R_{\mathrm{ct}} C_{\mathrm{dl}}$. In practical terms the double-layer characteristic frequency is situated $\omega_{\mathrm{dl}} / 2 \pi<1 \mathrm{kHz}$, because is usually observed that $R_{\mathrm{ct}}<1 \mathrm{k} \Omega \mathrm{cm}^{2}$. This means that establishing the polarization state at the interface takes place in times of the order of $\mathrm{ms}$ in many practical cases.

Apart from processes occurring at the interfaces between the electrolyte and the electrode material, independently of its porous or planar morphology, there is a set of circuit elements connected to the electrochemical response of the electrode bulk to the variation in the applied potential. The fundamental element accounts for the charging ability of any battery electrode, and it is represented by a capacitive mechanism labeled as chemical capacitance $C_{\mu} \cdot{ }^{2,3}$ It is defined as the ratio between the variation in electrode charge state produced by a change in the electrode potential $V$. Here the electrode potential is assimilated to the chemical potential of $\mathrm{Li}^{+}$in the electrode $\mu$, respect to the chemical

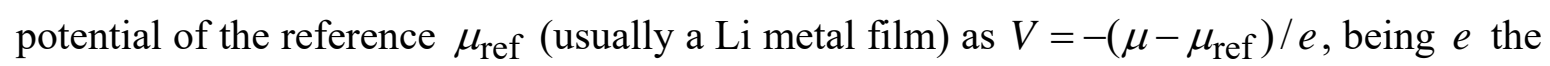
positive elementary charge. The actual concentration of Li-ions in the electrode bulk depends on the total concentration $N$ and the molar fraction in a given lithiated state $x$ as $n=N x$. In a battery electrode the ion chemical potential varies as a function of the amount of inserted charge $\mu(x)$, and this yields the steady-state charge-discharge curves. The chemical capacitance (per unit volume) can be then readily expressed as ${ }^{1}$

$$
C_{\mu}=e N \frac{d x}{d V}
$$

In equation $2, C_{\mu}$ has units of $\mathrm{F} \mathrm{cm}^{-3}$. Using the density of the active material, one can express it in units of $\mathrm{F} \mathrm{g}^{-1}$. The chemical capacitance is obviously related to the derivative of the discharge curve $-d Q / d V$ when very low charging rates are employed (quasiequilibrium charging). We note here that $C_{\mu}$ and $C_{\mathrm{dl}}$ are essentially different. The chemical capacitance measures the response to the variations in the chemical potential, while the double-layer capacitance is of dielectric nature, that is, it depends on the changes 
of the electrical field at the interface. As explained previously, electrochemical impedance experiments try to capture the electrical response to the perturbation of an electrode steady state. By definition, equation 2 gives information on the electrode low-frequency (long time) limit, which is ideally completely capacitive. In the highly lithiated state, battery electrodes always exhibit chemical capacitances in the range of $0.1-1 \mathrm{~F} \mathrm{~cm}^{-2}$, taking into account the effective electrode thickness, in such a way that $C_{\mu}>>C_{\mathrm{dl}}$.

Before the electrode attains the steady state, several rate-limiting mechanisms may operate that hinder or delay the charging. Similarly to that explained for the charge-transfer resistance, the transport of Li-ions inside the solid state bulk of the active material entails the crossing of local potential barriers between lattice sites. Concomitantly, ion transport is accompanied by energy loss, which in turn is electrically viewed as a resistance. This is the microscopic origin of the resistive mechanism behind the ion diffusion in the electrode material, which is called diffusion resistance $R_{\mathrm{d}}$. The driving force for $\mathrm{Li}^{+}$transport relates to the ion chemical potential gradient $\Delta \mu$ between adjacent sites. The combination of $R_{\mathrm{d}}$ and $C_{\mu}$ (diffusion and charging) yields the well-known diffusion impedance models as later addressed.

Diffusion resistance is not the only hindrance process for electrode charging. In electrode materials, the basic charging process involves the chemical reaction between $\mathrm{Li}^{+}$and the electrode constituents. For intercalation electrodes, the incorporation of ionic species does not excessively distort the lattice (topotactic intercalation). This implies that the reduction reaction itself is energetically favorable involving minor energy losses. On the contrary, for conversion or alloying electrodes $\mathrm{Li}^{+}$intake produces a full rearrangement of the host lattice structure with significant atomic displacement. In these last cases, the reaction itself, involving restructuring, kinetically governs the charging process. Again, reaction can be assimilated to an energy barrier surpass and represented by a resistive element, called reaction resistance $R_{\mathrm{r}}$. This resistance gathers information on the overall energy losses involved in the solid-state reaction, including atomic rearrangement. The rate-limiting mechanism can be determined by either $R_{\mathrm{d}}$ or $R_{\mathrm{r}}$ depending on the kinetically slowest process. The combination of $R_{\mathrm{r}}$ and $C_{\mu}$ (reaction and charging) also yields distinctive impedance responses. Either diffusion-limited or reaction-limited, these last circuit elements operate within the low-frequency range of the measuring frequency window $(\mathrm{Hz}-$ $\mathrm{mHz})$. 
Finally, there are in some cases minor contributions in the high-frequency range $(>1 \mathrm{kHz})$ originated by the response of external layers. The solid state interface (SEI) is commonly formed after cycling and introduces $R C$ subcircuits connected in series to the rest of the electrode equivalent circuit. The capacitive values are often in the range of $\mathrm{nF}-\mu \mathrm{F}$, because they are originated by dielectric responses of thicker films compared to the double-layer thickness.

\section{Basic impedance models}

The previously introduced circuit elements combine to make up the equivalent circuits used for fitting and data analysis of impedance responses in battery electrodes. A useful starting point is recalling the simplest Randle's circuit that gathers the main response mechanisms and simplifies hindrance processes. It is shown in the equivalent circuit of Figure 1a along with its corresponding impedance plot in the $Z^{\prime \prime}-Z^{\prime}$ complex plane. At high frequencies, the impedance experiment probes the interfacial processes that appear in a parallel combination and gives rise to a separate arc in the impedance plot. $R_{\mathrm{ct}}$ and $\omega_{\mathrm{dl}}$ can be directly read (from the real axis intercept and the maximum in $Z^{\prime \prime}$, respectively) and $C_{\mathrm{dl}}$ easily derived. The low-frequency response is dominated by the chemical capacitance because, in this simple circuit, all possible rate-limiting mechanisms in the bulk material are discarded. Although ideal, one can expect responses approaching this behavior when solid state diffusion restrictions disappear. It is noted here that battery electrodes rarely exhibit "ideal" capacitors accounting either for interfacial or bulk mechanisms. Instead, capacitance generalizations such as the well-known constant phase element (CPE) are used with impedance

$$
Z_{\mathrm{CPE}}=\frac{1}{Q(i \omega)^{n}}
$$

Here $i=\sqrt{-1}, \omega$ is the angular frequency of the perturbation, and $Q$ assimilates to the capacitance. CPE circuit elements should be considered as phenomenological descriptions of complex responses, in many cases connected to energetic or structural disorder. This element approaches pure capacitive responses in the case of $n=1$ with $Z_{C}=1 / i \omega C$. For $0.8 \leq n \leq 1$ one can consider that the impedance maintains the capacitive character. As observed in Figure 1b, CPE elements distort the high-frequency arc and yield inclined lowfrequency responses. 
The next step is the inclusion of solid state diffusion processes hindering the charging (capacitive) response of the electrode. Ion diffusion is an intricate mechanism that involves the transport of electronic carriers to assure the material electroneutrality. Fortunately, electronic movement is usually faster than the ionic one, at least for most of the battery electrodes of technological interest. This fact allows considering the diffusion of the ionic species (the slowest charge carrier) as the rate-limiting factor for electrode charging. In the simplest planar electrode structure, ions are incorporated from the electrolyte/electrode interface and progress into the material bulk. The model accounting for this mechanism is the so-called spatially-restricted diffusion model, which exhibits the so-called diffusion impedance, $Z_{\mathrm{D}}$ in Figure 1c. ${ }^{4}$ As aforementioned it assumes large enough electronic conductivity in such a way that $\mathrm{Li}^{+}$diffusion becomes the determining parameter of the charging process. Diffusion impedances undergo a pattern change at a certain characteristic frequency $\omega_{\mathrm{d}}$ at which a transition between a Warburg- to a capacitive-like behavior is observed. ${ }^{5,6}$ The frequency $\omega_{\mathrm{d}}$ is located near the elbow of the impedance plot in Figure $1 \mathrm{c}$, and relates to the ion chemical diffusion coefficient $D_{\mu}$ as

$$
\omega_{\mathrm{d}}=\frac{D_{\mu}}{L^{2}}
$$

Here $L$ corresponds to the diffusion length and related to the thickness of the electrode. The finite-length diffusion element is given by

$$
Z_{\mathrm{D}}=R_{\mathrm{d}}\left(\frac{i \omega}{\omega_{\mathrm{d}}}\right)^{-\gamma / 2} \operatorname{coth}\left[\left(\frac{i \omega}{\omega_{\mathrm{d}}}\right)^{-\gamma / 2}\right]
$$

$R_{\mathrm{d}}$ is the resistance associated with the ionic diffusion, and $\gamma$ relates to the deviation from the ideal spatially restricted diffusion impedance $(\gamma=1)$ in Figure 1c. The anomalous diffusion mechanism $(\gamma<1)$ in Figure $1 \mathrm{~d}$ is expected to occur in a multiphasic matrix. ${ }^{7}$ Diffusion of ions gives rise to distinctive impedance patterns characterized by Warburglike responses as $Z \propto(i \omega)^{-\gamma / 2}$ at intermediate frequencies. At lower frequencies the electrode charging is manifested in the capacitive response of the impedance through the chemical capacitance $C_{\mu}$. It is related to the characteristic diffusion frequency in equation 4 as

$$
\omega_{\mathrm{d}}=\frac{1}{R_{\mathrm{d}} C_{\mu}}
$$


The equivalent circuit of Figure $1 \mathrm{c}$ and $1 \mathrm{~d}$ includes the diffusion impedance model of equation 5 as an independent element $Z_{D}$ whose response varies in frequency in such a way that exhibits two different frequency limits. $Z_{D}$ is of general application for intercalation electrodes (topotactic insertion) in which it is well-established that the ion diffusion is the rate-limiting mechanism for electrode charging.

A different impedance model results from regarding that the charging process is limited by the solid-state reaction itself. That is to say that the rate-limiting mechanism is governed by microscopic processes occurring for the electrode material reduction different from the $\mathrm{Li}^{+}$ transport. The formulation of this kind of impedance models has deserved much less attention that the diffusion counterparts and only a few works have addressed the issue, particularly for conversion and alloying electrodes. ${ }^{8,}{ }^{9}$ Here the reduction reaction involves a large morphological restructuring, in many cases producing amorphous materials. As previously explained, one can gather all energy-loss processes occurring during the conversion reaction into an effective resistive element $R_{\mathrm{r}}$. It is known that reactions occur in an energetically and structurally complex environment that forces the spread of the reaction characteristic times (or frequencies). The characteristic time distribution caused by the inherent disorder can be incorporated into a generalization of the reaction resistance similarly to that used for generalizing capacitances. The CPE circuit element in equation 3 with an exponent $n=0$ can be viewed as a pure resistor. Therefore, a $Z_{C P E}$ with $0 \leq n \leq 0.3$ has been proposed for modeling the hindrance in conversion electrode charging. ${ }^{9}$ Hence, $R_{\mathrm{r}}$ connected in series with $C_{\mu}$ conveys the intuitive idea that the restriction for electrode charging takes place in the same current branch. This is specified in the impedance model of Figure 1e which gives rise to an impedance plot exhibiting a slow transition to the low-frequency capacitive limit. Some special cases exhibit a multistep conversion reaction, being the overall process modeled by several $R_{\mathrm{r}} C_{\mu}$ branches. ${ }^{9}$

Similarly to the diffusion characteristic frequency in equation 6 , one can define a characteristic reaction frequency $\omega_{\mathrm{r}}$ using the CPE expression in equation 3. $\omega_{\mathrm{r}}$ informs on the time scale of the conversion reaction as

$$
\omega_{\mathrm{r}}=\left(\frac{Q}{C_{\mu}}\right)^{\frac{1}{1-n}}
$$

The conversion frequency in equation 7 allows defining an effective conversion resistance 
accounting for the overall resistive contribution of the reaction from

$$
\omega_{\mathrm{r}}=\frac{1}{R_{\mathrm{r}} C_{\mu}}
$$

It is stressed that either diffusion- or reaction-limited the electrode kinetics can be represented by a well-defined characteristic frequency in equations 6 and 8 . In some relevant cases the distinction between both kinds of impedance models is not straightforward and mainly depends on the analysis of additional tests (electrode thickness or morphology variation, changes in electrolyte composition...) that might assist in the model selection. In other cases, both diffusion and restructuring contribute to the kinetic limitation, being the separation of their individual influences unachievable in terms of well-distinguished characteristic frequencies. Here, the estimation of a response frequency and effective hindrance resistance can be made using the model in Figure 1e, which suffices as a phenomenological approach for many practical purposes.

In some experiments, intermediate-frequency distortions appear involving an arc just before the low-frequency capacitive behavior governed by $C_{\mu}$. Previous analyses connected these trends to distributions of diffusion lengths, ${ }^{10}$ or electronic transport limitations. ${ }^{11}$ It can be also linked with an extra chemical capacitance $C_{\mu}^{n}$ accounting for the presence into the storage material of "free" $\mathrm{Li}^{+}$ions. These ions occupy sites in the host lattice that do not belong to kinetically stable charging locations, which give rise the defined $C_{\mu}$. A theoretical model was previously introduced. ${ }^{8}$ Figure $1 \mathrm{f}$ shows the proposed equivalent circuit, including $C_{\mu}^{n}$, along with an example of the impedance plot.

So far we have been dealing with of the impedance response of compact electrodes. If the electrolyte penetrates the electrode and wets the active particles the effective diffusion length in the solid-state may be significantly reduced $L_{\mathrm{eff}}<<L$, in comparison to the electrode thickness giving rise to large overestimations in $D_{\mu}$. The so-called porous impedance model allows addressing these last cases. ${ }^{10-12}$ Hence, it is a matter of experimental check to discern if $L_{\text {eff }} \approx L$, in such a way that the finite-length diffusion mechanism in equation 4 can be consistently used to determine $D_{\mu}$. The porous models rely on the formation of an extended double-layer in the vicinity of the active particles inside the electrode matrix. It is expected then that larger values for $C_{\mathrm{dl}}$ are obtained. It should be also noted that in some instances, the sole analysis of the impedance response is not often enough to decide about the compact or porous character of the electrode and additional tests are needed here to distinguish about. 
In the following, we address the electrochemical response of several electrode materials in the light of the previously described impedance models. Attention will be paid to the kinetics mechanisms to illustrate the use of the models, rather than detailing the charge/discharge profiling and electrode general performance. 
(a)

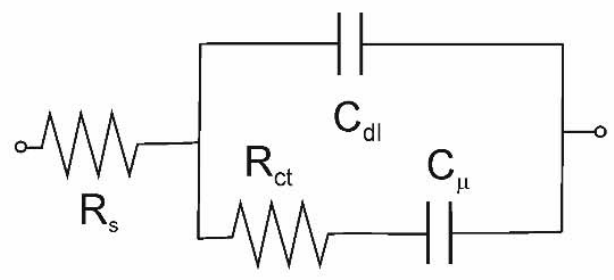

(b)

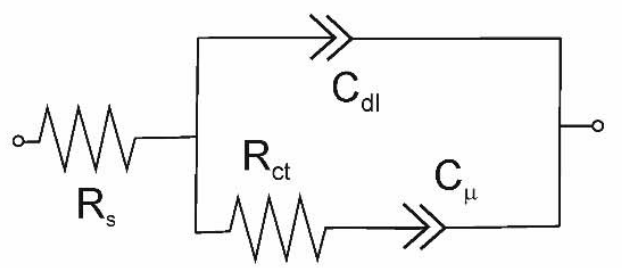

(c)
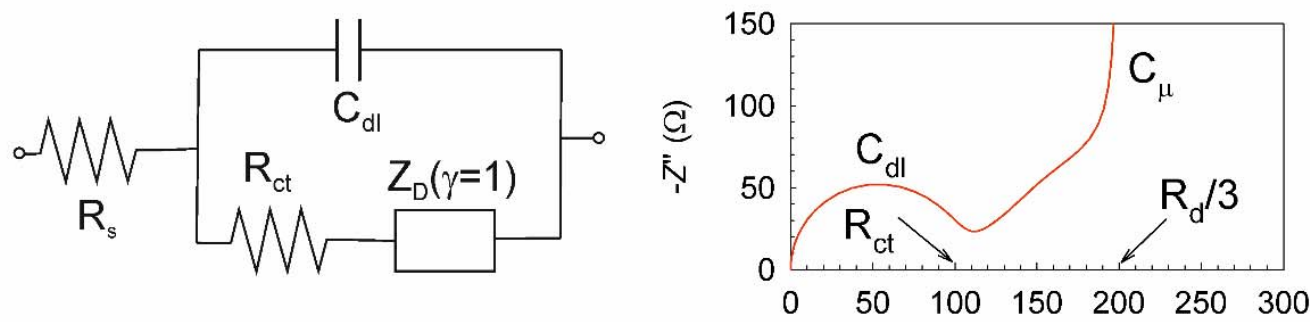

(d)
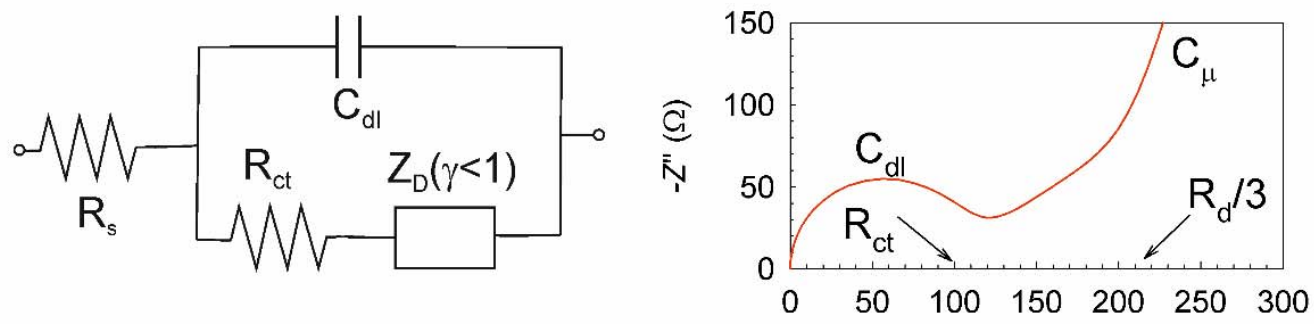

(e)
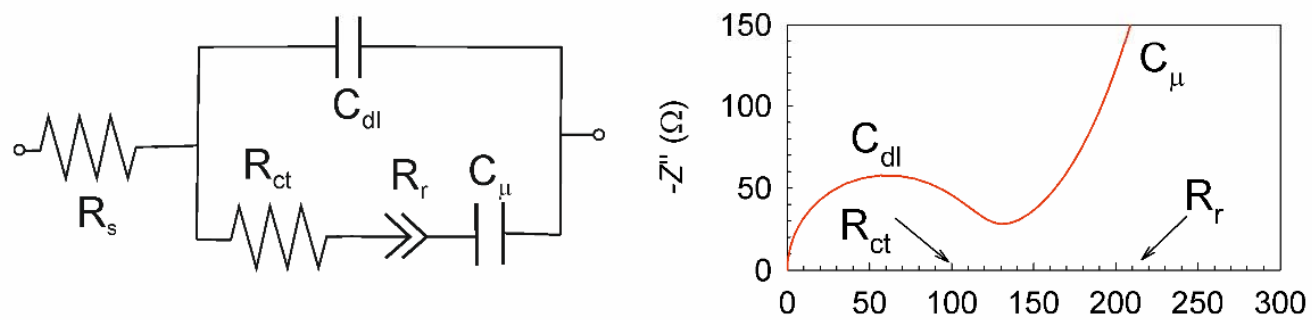

(f)

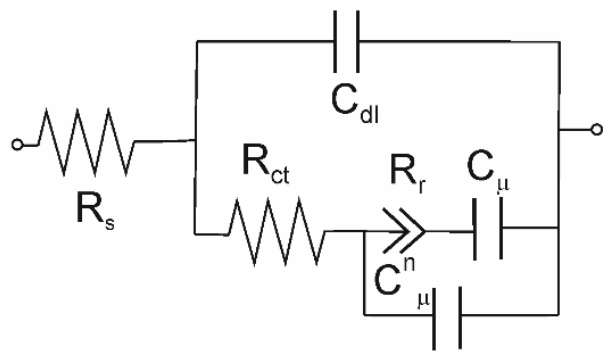

Figure 1. Equivalent circuits and their corresponding impedance plot $\left(Z^{\prime \prime}-Z^{\prime}\right.$ complex 
plane) for different battery electrode mechanisms. $R_{\mathrm{S}}$ accounts for the solution resistance. (a) Ideal Randle's circuit without any solid-state charging limitation. (b) After incorporating constant-phase elements (CPE) in substitution of pure capacitances. (c) Ideal spatially-limited diffusion model $Z_{\mathrm{D}}$ with disorder parameter $\gamma=1$. (d) Anomalous spatially-limited diffusion model $Z_{\mathrm{D}}$ with disorder parameter $\gamma<1$. (e) Reaction-limited model including the generalization of reaction resistance. In all the plots: $C_{\mathrm{dl}}=1 \mu \mathrm{F}$, $C_{\mu}=1 \mathrm{mF}$ and $R_{\mathrm{ct}}=100 \Omega$. (b) $n=0.85$ for both capacitances. (c) $R_{\mathrm{d}}=300 \Omega$, and $\gamma=1$. (d) $R_{\mathrm{d}}=300 \Omega$, and $\gamma=0.85$. (e) $R_{\mathrm{r}}$ calculated using $Q=0.005 \Omega^{-1}$ (is) ${ }^{-n}$ and $n=0.2$ in equation 3. (f) Including $C_{\mu}^{n}=100 \mu \mathrm{F}$.

\section{Cathodes: $\mathrm{Li}\left[\mathrm{Ni}_{y} \mathrm{Co}_{z} \mathrm{Mn}_{1-y-z}\right] \mathrm{O}_{2}$ and $\mathrm{LiFePO}_{4}$.}

To start with we discuss the impedance response of two kinds of actively studied cathode materials: the layered oxide $\mathrm{Li}\left[\mathrm{Ni}_{0.8} \mathrm{Co}_{0.06} \mathrm{Mn}_{0.14}\right] \mathrm{O}_{2}{ }^{13}$ and the olivine $\mathrm{LiFePO}_{4}{ }^{14}$ In the former the transition metal (TM) is linked directly to the $\mathrm{O}$ atom, while in the latter the TM is bonded to a polyanion. In consequence, the $\mathrm{O}$ in the TM oxides show high nucleophilic character that can rapidly react with the electrophilic alkyl carbonate molecules and acidic species formed from the decomposition of salt during the cycling of the battery. The presence of covalently bonded $\mathrm{PO}_{4}$ units in the case of $\mathrm{LiFePO}_{4}$ leads to good structural and chemical stabilities without the liberation of oxygen. On the other hand, the different $\mathrm{Li}$ arrangement in both cathodes (interconnected edge-shared $\mathrm{LiO}_{6}$ octahedral sites in the layered oxide while half in the case of the olivine) provides fast two-dimensional Li ion diffusion $\left(\sigma_{\mathrm{Li}}\right)$ in $\mathrm{Li}\left[\mathrm{Ni}_{0.8} \mathrm{Co}_{0.06} \mathrm{Mn}_{0.14}\right] \mathrm{O}_{2}$ and slow one-dimensional $\sigma_{\mathrm{Li}}$ in $\mathrm{LiFePO}_{4}{ }^{15}$ Also, the electrical conductivity $\left(\sigma_{\mathrm{e}}\right)$ in the former is higher than in the latter. In consequence, $\mathrm{LiFePO}_{4}$ shows low rate capability compared to $\mathrm{Li}\left[\mathrm{Ni}{ }_{0} \mathrm{Co}_{0.06} \mathrm{Mn}_{0.14}\right] \mathrm{O}_{2}$. These features are reflected in the parameters determined by experimental EIS measurements and analyzed with the equivalent circuit modeling.

For both cathodes, $\mathrm{Li}\left[\mathrm{Ni}_{0.8} \mathrm{Co}_{0.06} \mathrm{Mn}_{0.14}\right] \mathrm{O}_{2}$ and $\mathrm{LiFePO}_{4}$, the same equivalent circuit model can be applied, which corresponds to the kind drawn in Figure 1f, with the additional inclusion of a high-frequency parallel $R_{\mathrm{sf}} C_{\mathrm{sf}}$ subcircuit. In this model, $R_{\mathrm{sf}}$ and $C_{\mathrm{sf}}$ yield an arc related to the resistance and capacity of the Li ions rapid diffusion through the solid film (SF) formed at the cathode-electrolyte interface due to the decomposition reactions during lithiation-delithiation processes. ${ }^{13,14}$ The use of the model in Figure $1 \mathrm{f}$ is adopted here as a phenomenological approach in the case of multiparticle electrodes even with rate- 
limiting mechanism dominated by ion diffusion. It allows extracting the influence of hindrance processes and compare electrode performances.

In the case of $\mathrm{Li}\left[\mathrm{Ni} 0.8 \mathrm{Co} 0.06 \mathrm{Mn}_{0.14}\right] \mathrm{O}_{2}$, the two subcircuits (interface- and bulk-originated) in Figure 1f and the additional high-frequency contribution can be clearly identified in the EIS spectra, shown in Figure 2a. The two arcs at high frequency are related to the interfacial processes (resistances assigned to the SF and charge transfer) and that at low frequencies assigned to the chemical energy storage mechanism. In particular, Figure 2a shows the EIS spectra registered at $3.9 \mathrm{~V}$ during lithiation process of the cathodes of general formula $\mathrm{Li}\left[\mathrm{Ni}_{0.8} \mathrm{Co}_{0.06} \mathrm{Mn}_{0.14}\right] \mathrm{O}_{2}$, but with different atomic distribution: conventional composition (CC) and two-sloped full concentration gradient (TSFCG). ${ }^{13}$ The core in TSFCG is rich in $\mathrm{Ni}$ (cation responsible of high capacitance) while the concentration of $\mathrm{Mn}$ increases on the surface layer of composition $\mathrm{Li}\left[\mathrm{Ni}_{0.64} \mathrm{Co}_{0.06} \mathrm{Mn}_{0.30}\right] \mathrm{O}_{2}$. Among the three $\mathrm{TM}, \mathrm{Ni}$ is reported to enhance the nucleophilicity of the $\mathrm{O}$, and is more surface reactive than the other two species (Co and $\mathrm{Mn}){ }^{16}$ Then, the lower concentration of $\mathrm{Ni}$ in the surface layer of TSFCG structure reduces the reactivity of the cathode with the electrolyte and, in consequence, the resistance related to the SF in one order of magnitude lower. The formation of SF affects the capacity fade, while TSFCG shows capacity retention of $\sim 90 \%$ after 100 cycles, for CC is $\sim 78 \%$.
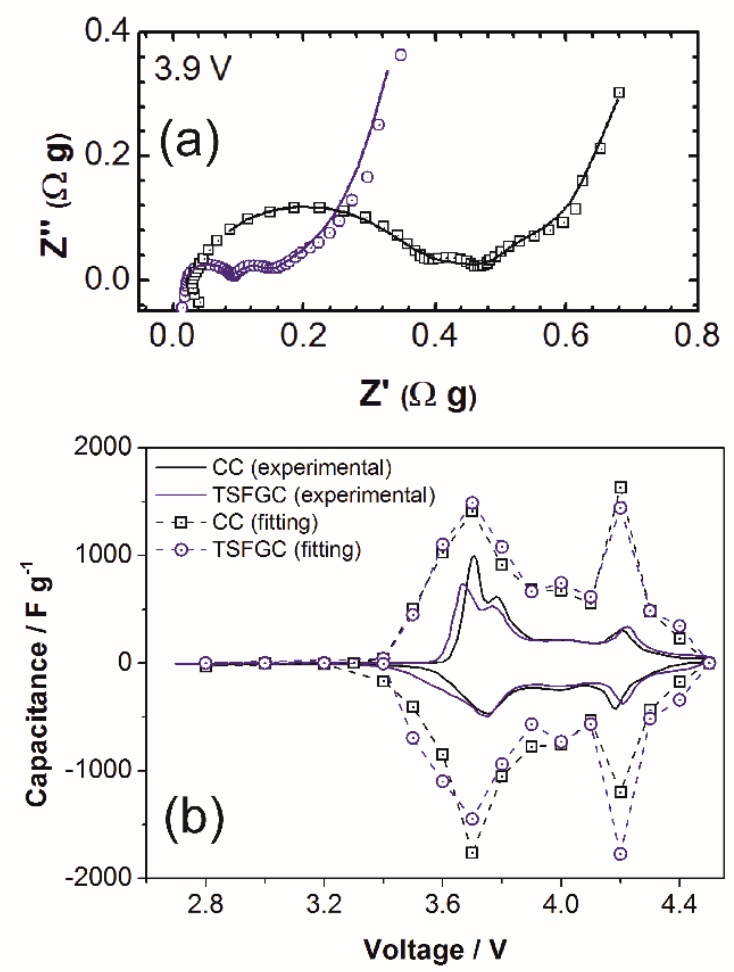
Figure 2. (a) Impedance plots at $3.9 \mathrm{~V}$ during lithiation process in TSFCG and CC positive electrodes. The experimental data are represented by points and the fitting with solid lines. (b) Chemical capacitance of the CC and TSFCG cathodes obtained from $-d Q / d V$ of the charge/discharge measurements at $0.1 \mathrm{C}$, and $C_{\mu}$ calculated from the EIS data. Reproduced (adapted) from Ref. ${ }^{13}$ with permission from the Royal Society of Chemistry.

EIS measurements also show that the main operating difference between CC and TSFCG electrodes is kinetic and not thermodynamic. This effect is clearly observed through the determination of $C_{\mu}$ from EIS, which theoretically corresponds to the differential charge change in the electrode upon voltage variation in quasi-equilibrium state $-d Q / d V$, calculated from the measurements of the charge-discharge curve. $C_{\mu}$ values determined from the equivalent circuit model (Figure 1f) and charge-discharge measurements are provided in Figure $2 \mathrm{~b}$. From both methods, the capacitance show the typical shape of this material associated with the overall reaction $\mathrm{Li}_{x}\left(\mathrm{Ni}_{y}{ }^{2+} \mathrm{Co}_{z}{ }^{3+} \mathrm{Mn}_{1-y-z}{ }^{4+}\right) \mathrm{O}_{2} \leftrightarrow\left(\mathrm{Ni}_{y}{ }^{2+} \mathrm{Co}_{z}{ }^{3+} \mathrm{Mn}_{1-}\right.$ $\left.y-{ }^{4+}\right) \mathrm{O}_{2}+x \mathrm{Li}^{+}+x \mathrm{e}^{-}$. The larger values extracted from EIS measurements in comparison to galvanostatic experiments are caused by the different experimental conditions: the former are extrapolated from low-frequency limit (quasi steady-state) while the latter cannot be regarded as a true steady-state even at the low current rate of $0.1 \mathrm{C}$. More interesting is the fact that in dynamic measurements (charge-discharge and cyclic voltammetry measurements) the peak at $4.2 \mathrm{~V}$ (ascribed either to the Co oxidation state change or oxide phase transition) is lower than at $3.7 \mathrm{~V}$ (assigned to the change in the oxidation state of Ni). ${ }^{17,} 18$ Meanwhile, $C_{\mu}$ values obtained from EIS are slightly higher for the peak registered at $4.2 \mathrm{~V}$. This fact together with the similarity in $C_{\mu}$ values from EIS for both $\mathrm{CC}$ and TSFCG electrodes point out that the lithiation/delithiation processes are kinetically rather than thermodynamically inhibited in the studied cathodes.

While EIS show the critical formation of SF films in $\mathrm{Li}\left[\mathrm{Ni}{ }_{0.8} \mathrm{Co}_{0.06} \mathrm{Mn}_{0.14}\right] \mathrm{O}_{2}$ cathodes, this detrimental film is not (or scarcely) formed in the family of $\mathrm{LiFePO}_{4}$ positive electrodes. The low reactivity of $\mathrm{LiFePO}_{4}$ cathodes with the electrolyte is observed in Figure 3a, where the arc at high frequency related to the SF cannot be observed at all. In this case, EIS show two patterns: a flattened arc at high frequencies linked to the interface-related $R_{\mathrm{ct}} C_{\mathrm{dl}}$ subcircuit, and a capacitive behaviour associated with the Li ion storage inside the cathode, manifested by $C_{\mu}$.

Two different strategies to increase the Li ion diffusion and electronic conductivity of $\mathrm{LiFePO}_{4}$ (LFP) were tested by giving molecular wiring with: carbon (C-LFP) and 
PEDOT:PSS (LFP/PEDOT(blend)). ${ }^{14}$ The two resistances $\left(R_{\mathrm{ct}}\right.$ and $\left.R_{\mathrm{r}}\right)$ that hindrance lithiation/delithiation processes in these cathodes can be estimated by means of the equivalent circuit model, shown in Figure 1f. The estimated values for the lithiation process are shown in Figure 3b, which shows that PEDOT:PSS reduces the charge transfer resistance more effectively than C, practically by one order the magnitude above $3.4 \mathrm{~V}$. ${ }^{19}$ Below this voltage, $R_{\mathrm{ct}}$ of the LFP/PEDOT(blend) electrode starts increasing, and at 2.2 $\mathrm{V}$ both cathodes show similar values. Also, lower $R_{\mathrm{r}}$ values are obtained for LFP/PEDOT(blend) than for C-LFP, indicating that the introduction of the electric molecular wiring also influence the lithiation reaction resistance of the phosphate matrix. In this regard, the use of PEDOT(blend) is an effective strategy to reduce the resistances that govern LFP lithiation/delithiation processes due to the high conductivity of both electrons and ions of the polymer, ${ }^{20}$ and the good embedded structure of the LFP nanoparticles in the PEDOT matrix. This is reflected in the charge-discharge plots of LFP/PEDOT(blend) and C-LFP cathodes, Figure $3 \mathrm{c}$ and d. At $0.1 \mathrm{C}$, both cathodes show similar capacity and low hysteresis, but with the increase of the charge-discharge rate, the hysteresis effect is more noticeable in the C-LFP cathode at the same time that the capacity decreases. 

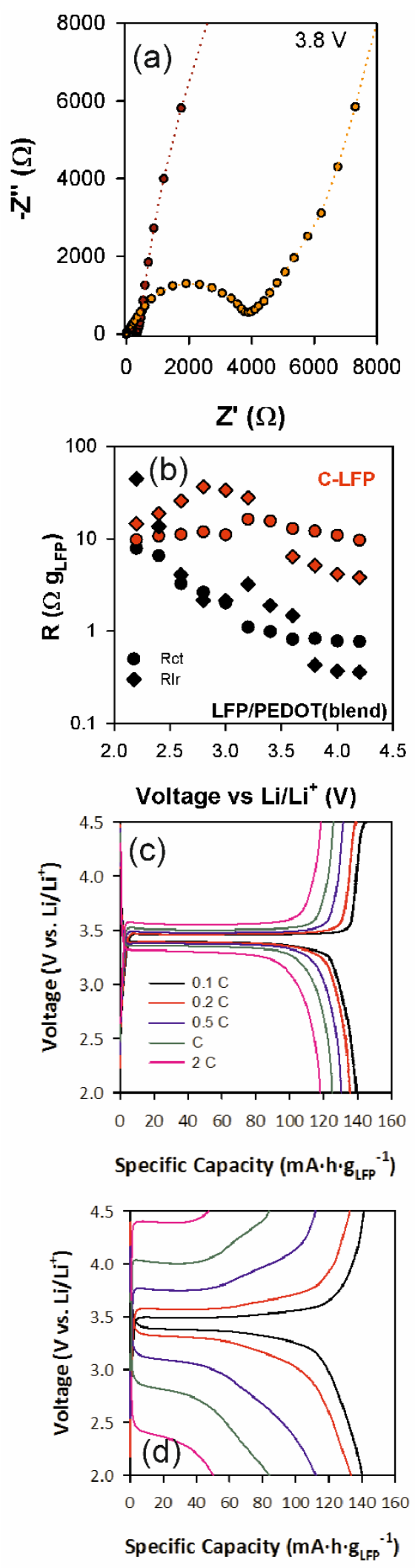

Figure 3. (a) Impedance plots of measured (dots) and fitting results (lines) at $3.8 \mathrm{~V}$ of discharge for: LFP/PEDOT(blend) (red dots) and C-LFP (yellow dots). (b) Fitting parameters in discharge process for LFP(blend) (solid symbols) and C-LFP (hollow symbols): $R_{\mathrm{ct}}$ is symbolized by circles and $R_{\mathrm{lr}}$ by rhombus. Charge-discharge curves at 
different rates for: (c) LFP/PEDOT(blend) and (d) C-LFP. Reproduced (adapted) from ref. ${ }^{14}$ with permission from Elsevier.

\section{Anodes: MWCT@ $\mathrm{TiO}_{2}$ and $\mathrm{MAPbBr}_{3}$.}

To improve the handicaps that materials for lithium ion battery (LIB) anodes present at high cycling rates, such as low $\mathrm{Li}^{+}$diffusion, scarce electron transport and high resistances at the electrode/electrolyte interfaces, new approaches and new materials are studied. Combining different materials allows creating composites with superior properties than the pristine counterparts. Multiwalled carbon nanotube and $\mathrm{TiO}_{2} \quad\left(\mathrm{MWCNT} @ \mathrm{TiO}_{2}\right)$ composites as anodes achieved to improve the electronic transport offered by the carbon, the reversibility for $\mathrm{Li}^{+}$ion insertion and mechanical stability provided by $\mathrm{TiO}_{2}$. Also, a specific capacity as high as $250 \mathrm{~mA} \mathrm{~h} \mathrm{~g}^{-1}$ is delivered, which doubles that encountered for $\mathrm{TiO}_{2}$-based anodes. ${ }^{21}$ Impedance analysis typically shows two patterns with distinguishable time constants associated to interfacial and charging mechanisms. As it is shown in Figure 4, the composite (MWCNT@ $\mathrm{TiO}_{2}$ ) exhibits resistive components connected to transport of $\mathrm{Li}^{+}$ion before reaching stable sites inside the active matrix.

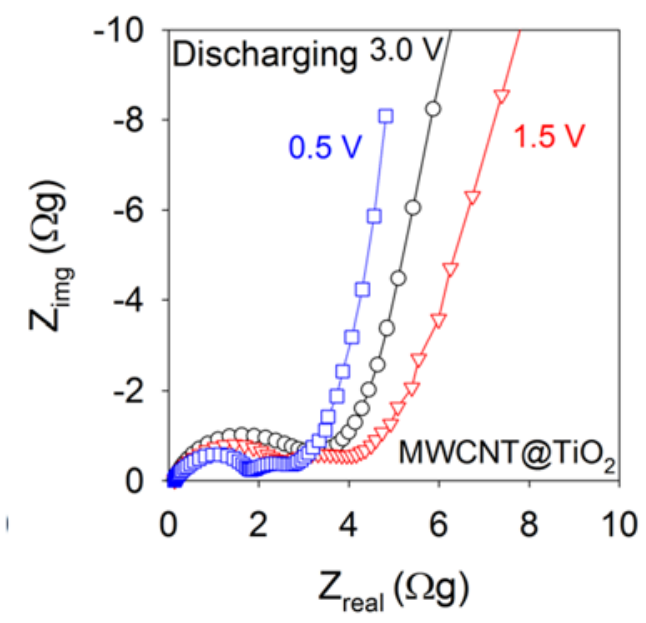

Figure 4 Impedance response of MWCNT@ $\mathrm{TiO}_{2}$ electrodes at different discharge potentials. Reproduced (adapted) from ref. ${ }^{21}$ with permission from Elsevier.

In the case of pure $\mathrm{TiO}_{2}$-electrode at high voltage, $R_{\mathrm{ct}}$ is so high that it does not allow detecting other process within the measuring frequency range. According to Figure 1f, the low-frequency part of the spectra conveys information on $R_{\mathrm{r}}$ (lithiation reaction) and $C_{\mu}$ (chemical capacitance). A summary of the parameters extracted from fitting, during charge and discharge in potentiostatic mode is depicted in Figure 5. 

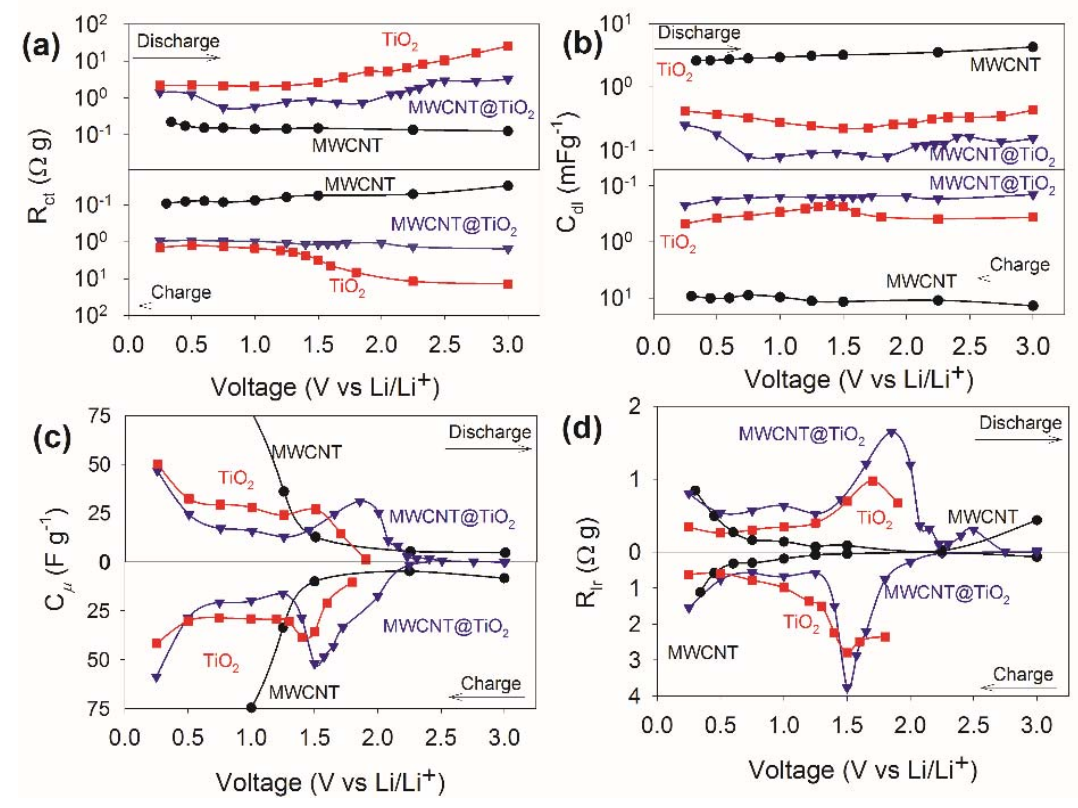

Figure 5 Parameters derived from fitting of EIS data for $\mathrm{TiO}_{2}$-based electrodes: (a) Charge transfer resistance $R_{\mathrm{ct}}$, (b) double layer capacitance $C_{\mathrm{dl}}$, (c) chemical capacitance, $C_{\mu}$, and (d) lithiation-reaction resistance $R_{\mathrm{r}}$. Reproduced (adapted) from ref. ${ }^{21}$ with permission from Elsevier.

Firstly, the high-frequency elements $R_{\mathrm{ct}}$ and $C_{\mathrm{dl}}$ (Figure $5 \mathrm{a}$ and $5 \mathrm{~b}$ ) exhibit rather voltage-independent values for electrodes comprising MWCNT, with and without $\mathrm{TiO}_{2}$. Although at lower potentials both $\mathrm{TiO}_{2}$ and MWCNT@TiO 2 are able to insert $\mathrm{Li}^{+}$ions with similar (diminished) hindrance, when the voltage increases over $1.5 \mathrm{~V}$, a much larger charge transfer resistance is observed for pristine $\mathrm{TiO}_{2}(\sim 10 \Omega \mathrm{g})$ compared to MWCNT@TiO, which keeps it at $\sim 1 \Omega \mathrm{g}$. It is known that $R_{\mathrm{ct}}$ is directly related to the electronic conductivity of the host material, ${ }^{22}$ because conductive hosts facilitate the $\mathrm{Li}^{+}$ ion to overcome the potential barrier appearing at the electrolyte/semiconductor interface, in such a way that MWCNTs assist the $\mathrm{Li}^{+}$ion incorporation into the $\mathrm{TiO}_{2}$ matrix through the formation of Ti-C bonds. ${ }^{21}$

The chemical capacitance $C_{\mu}$ in Figure 5c exhibits well-defined peaks both for MWCNT@ $\mathrm{TiO}_{2}$ and $\mathrm{TiO}_{2}$ electrodes in the lithiation-reaction voltage range, with comparable values around $250 \mathrm{~F} \mathrm{~g}^{-1}$. The resistance accompanying the lithiation reaction $R_{\mathrm{r}}$ shows also a similar behavior giving values of 1-3 $\Omega \mathrm{g}$ (Figure $5 \mathrm{~d}$ ). Differences of a factor two are observed in $R_{\mathrm{r}}$ between MWCNT@ $\mathrm{TiO}_{2}$ and $\mathrm{TiO}_{2}$ electrodes probably related to the strain in $\mathrm{TiO}_{2}$ particles caused by the interface $\mathrm{MWCNT} / \mathrm{TiO}_{2}$, which slightly modifies the lithiation reaction kinetics. Therefore, $\mathrm{TiO}_{2}$ and $\mathrm{MWCNT} @ \mathrm{TiO}_{2}$ exhibit 
comparable specific capacity, but the charge transfer resistance for the latter is reduced by a factor 10 , implying a key role of MWCNT to favor the interfacial $\mathrm{Li}^{+}$ion intake from the electrolyte. This is the main mechanism that explains the superior rate capability (power performance) observed of MWCNT@ $\mathrm{TiO}_{2}$ in comparison to $\mathrm{TiO}_{2}$ electrodes.

On the other hand, recently hybrid halide perovskites have been reported as promising charge-storage materials for lithium-ion batteries anodes. ${ }^{23,} 24$ These compounds own interesting electronic and photonic properties, and ionic migration allows for a variety of applications in electrochemical devices. The hybrid perovskite $\mathrm{CH}_{3} \mathrm{NH}_{3} \mathrm{PbBr}_{3}$ has been utilized as active material for the intercalation anode, which behaves as a compact structure in which the dimensionality of $\mathrm{Li}^{+}$transport is $3 \mathrm{D}$. As the perovskite lithiation progresses, specific capacity values as high as $400 \mathrm{~mA} \mathrm{~h} \mathrm{~g}^{-1}$ are reached (Figure 6a), which implies a Li-ion concentration as high as $10^{21} \mathrm{~cm}^{-3}$, given perovskite densities approximately equal to $4.16 \mathrm{~g} \mathrm{~cm}^{-3}$. The host matrix becomes fully lithiated at potentials below $0.5 \mathrm{~V}$ vs. $\mathrm{Li} / \mathrm{Li}^{+}{ }^{23}$ Chemical diffusion coefficient of lithium ion within the perovskite lattice exhibits values as high as $D_{\mu} \approx 10^{-7} \mathrm{~cm}^{2} \mathrm{~s}^{-1}$, which implies ionic conductivities within the range of $10^{-3} \Omega^{-1}$ for highly lithiated electrodes. The impedance plots (Figure $6 \mathrm{~b}$ ) can be modeled by the equivalent circuit in Figure $1 \mathrm{~d}$. The intermediate- and low-frequency impedance response exhibits clearly a diffusive-capacitive behavior that depends on the voltage (charging) state. Diffusion impedances undergo a pattern change at a certain characteristic frequency $\omega_{\mathrm{d}}$ (Equation 4 and 6 ) at which a transition between a Warburg- to a capacitivelike behavior is observed. The superionic property of organohalide perovskites can be exploited in applications and devices in which fast ionic migration is an essential requirement. 

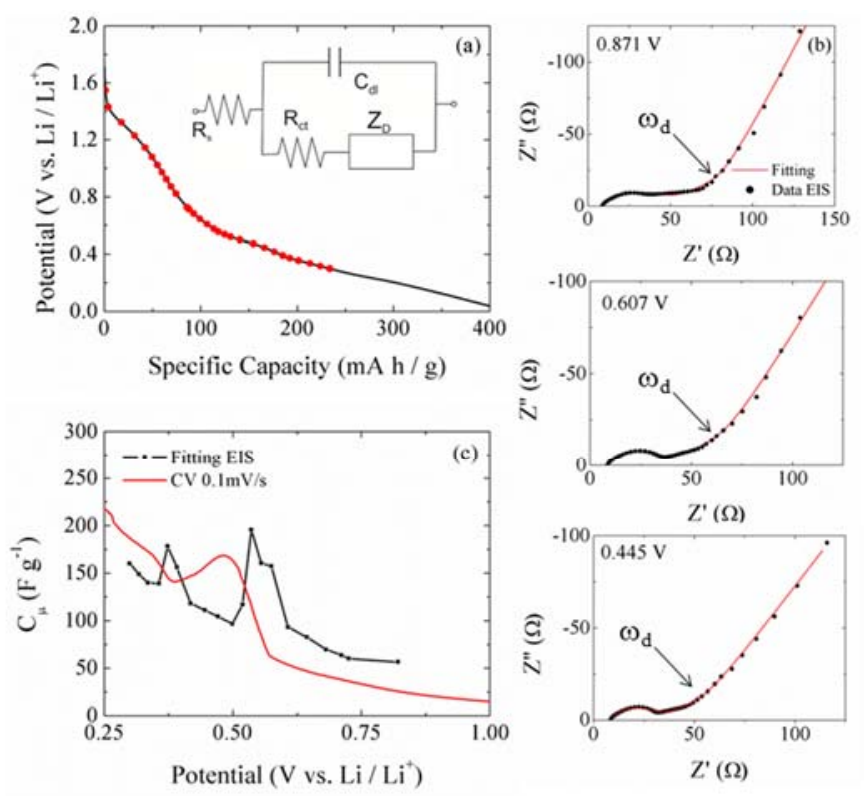

Figure 6 (a) Selected potentials and charge state of the electrode lithiation process for insitu EIS analysis during the discharge process of $\mathrm{CH}_{3} \mathrm{NH}_{3} \mathrm{PbBr}_{3}$ anodes. Inset: equivalent circuit used (Figure 1d). (b) Impedance spectroscopy response at different steady-state voltages. Solid line corresponds to fits using the equivalent circuit. The diffusion response frequency $\omega_{\mathrm{d}}$ is marked. (c) $C_{\mu}$ calculated from different data: EIS and cyclic voltammetry as $-d Q / d V$ plotted vs. potential during discharge process. Reproduced from ref. ${ }^{24}$ with permission from WILEY-VCH.

\section{Conversion and alloying anodes: $\mathrm{FeOOH}, \mathrm{ZnFe}_{2} \mathrm{O}_{4}$ and $\mathrm{Si} / \mathrm{Ge}$.}

In this section, a review about the use of the impedance technique for analyzing conversion and alloying anodes is done. Three of the more promising alternatives to carbon anodes were characterized: conversion-reaction of amorphous iron oxyhydroxide nanosheets $(\mathrm{FeOOH})$ origins a $R C$ series subcircuit that governs the hysteresis behavior; $\mathrm{Si} / \mathrm{Ge}$ doubled layered nanotube anodes ( $\mathrm{Si} / \mathrm{Ge} \mathrm{DLNT})$ in which Ge shell plays a role as an electron supplier; and carbon-coated zinc ferrite anodes $\left(\mathrm{ZnFe}_{2} \mathrm{O}_{4}-\mathrm{C}\right)$ where impedance study allows for the quantification of the kinetic parameters governing the various lithiation steps. For the two first types of anodes the equivalent circuit showed in Figure $1 \mathrm{f}$ is employed, whereas in the case of the $\mathrm{ZnFe}_{2} \mathrm{O}_{4}-\mathrm{C}$ anodes a new mechanism at high frequencies must be taken into account incorporating a new branch in the proposed equivalent circuit, as it is explained below. 

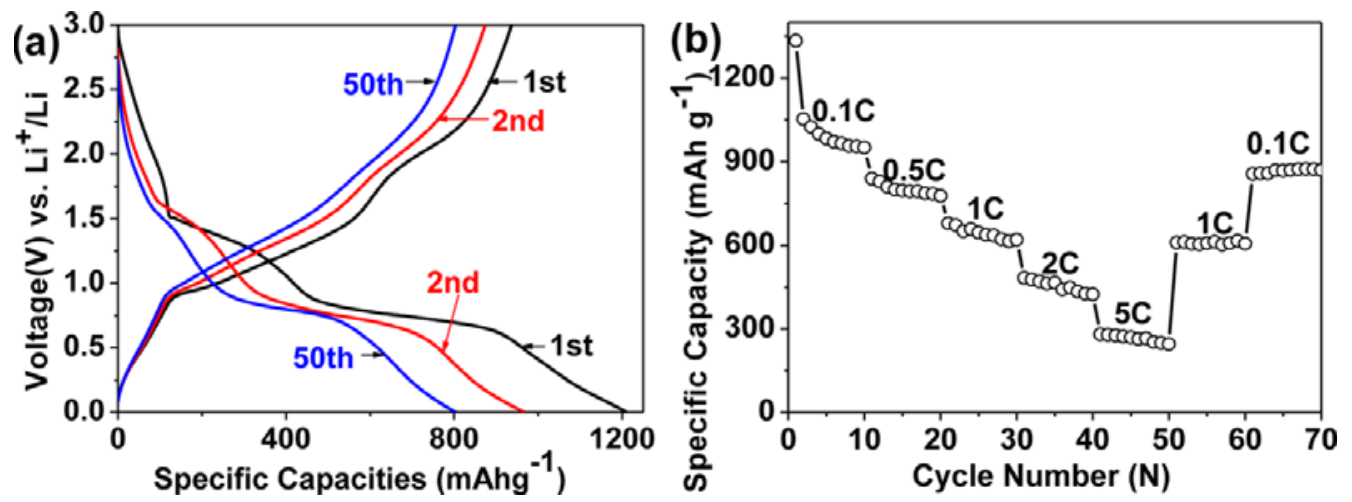

Figure 7 (a) Charge-discharge voltage profiles of the 1st, 2nd and 50th cycle at a current density of $0.11 \mathrm{C}\left(100 \mathrm{~mA} \mathrm{~g}^{-1}\right)$. (b) Cycling performance at different $\mathrm{C}$ rates. Reproduced (adapted) from ref. ${ }^{8}$ with permission (2013) from American Chemical Society.

Amorphous $\mathrm{FeOOH}$ anodes present a highly porous nanostructure and high surface area $\left(223 \mathrm{~m}^{2} \mathrm{~g}^{-1}\right) .{ }^{8}$ In the Figure $7 \mathrm{a}$, the first discharge cycle shows at $0.95 \mathrm{~V}$ the specific capacity is $437 \mathrm{mAh} \mathrm{g}^{-1}$, which corresponds to incorporation of approximately $1.45 \mathrm{~mol}$ of $\mathrm{Li}$ per mol of FeOOH. The specific capacity of $914 \mathrm{~mA} \mathrm{~h} \mathrm{~g}^{-1}$ corresponds to the insertion of approximately insertion of $3 \mathrm{~mol} \mathrm{Li}$ per $\mathrm{mol}$ of $\mathrm{FeOOH}$ at discharge potential of $0.5 \mathrm{~V}$, implying complete conversion reaction between $\mathrm{Li}$ and $\mathrm{FeOOH}$ with the formation of elemental $\mathrm{Fe}$ and $\mathrm{Li}_{2} \mathrm{O}\left(\mathrm{FeOOH}+3 \mathrm{Li}^{+}+3 \mathrm{e}^{-} \rightarrow \mathrm{Fe}+\mathrm{Li}_{2} \mathrm{O}+\mathrm{LiOH}\right)$. Electrodes also show good rate capability with a discharge capacity as high as $642 \mathrm{~mA} \mathrm{~h} \mathrm{~g}^{-1}$ at $1 \mathrm{C}$ (Figure $7 \mathrm{~b}$ ). EIS analysis of amorphous FeOOH electrode at different states of discharge (SOD) and states of charge (SOC) has been studied upon the conversion reaction is expected to be reversible and stabilized afterward. This type of electrodes undergo an overall material rearrangement in both chemical and structural nature lead us to regard the conversion reaction as the rate-limiting process of the change in electrode state of charge/discharge. The equivalent circuit in Figure 1f allows for full analysis of the kinetic response. 
(a)

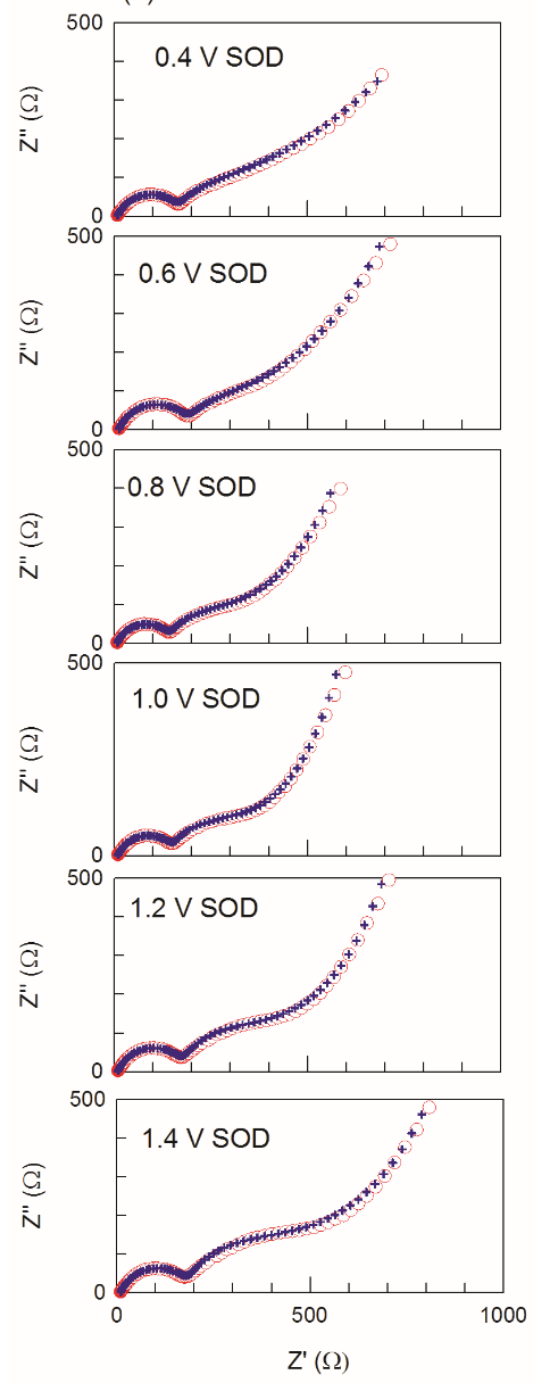

(b)
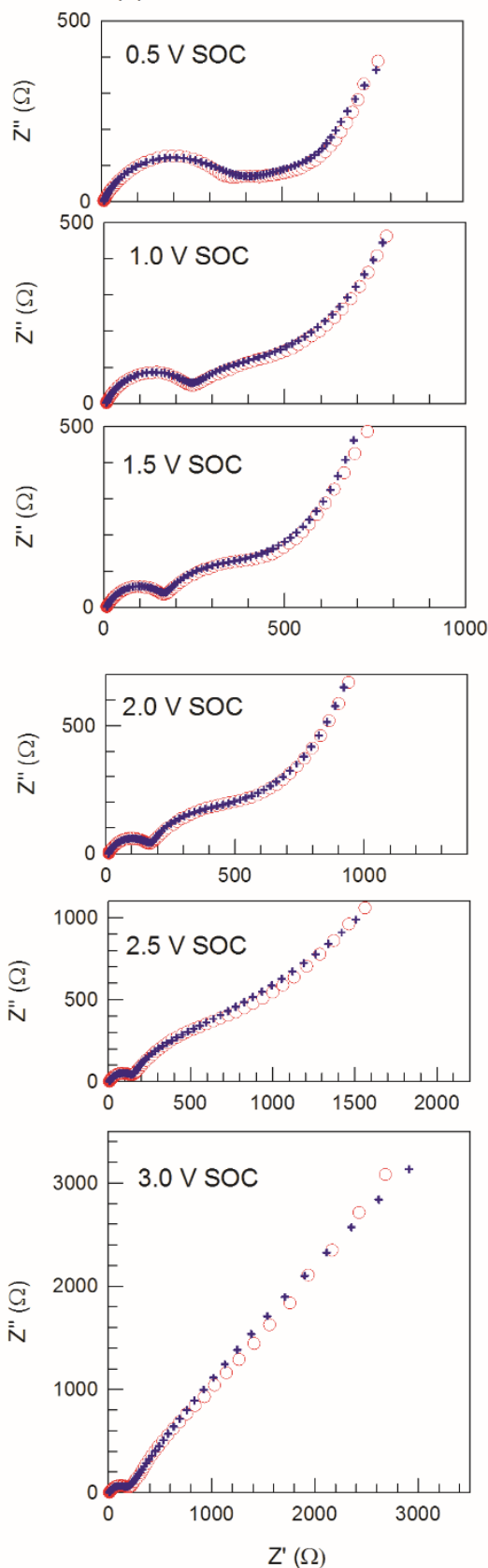

Figure 8 Impedance response of amorphous FeOOH anodes measured at different voltages corresponding either to (a) discharge regime SOD or (b) charge regime SOC. Experimental data (dot) and fits (cross) are displayed for comparison. Reproduced (adapted) from ref. ${ }^{8}$ with permission (2013) from American Chemical Society.

The electrical parameters at different SOD and SOC have also been calculated from fitting in Figure 8 . The conversion reaction resistance $R_{\mathrm{r}} \approx 500 \Omega$ results greater than $R_{\mathrm{ct}}$, which 
implies that the conversion reaction limits the electrode charging. The conversion-reaction kinetics can be alternatively accessed by examining the response frequency. Values of $\omega_{\mathrm{r}}$ (Equation 8) below $0.04 \mathrm{~s}^{-1}$ are found. ${ }^{8}$ Its maximum is located within the potential interval of the major conversion reaction according to the discharge voltage profile. Voltage shift in $\omega_{\mathrm{r}}$ of $\sim 0.5 \mathrm{~V}$ is noticed between discharge and charge regimes. This fact points to the hysteretic behavior of the conversion reaction. ${ }^{25}$ Therefore, EIS analysis reproduces the hysteresis observed between charge and discharge profiles in Figure 7a. Accordingly, one can conclude that the conversion-reaction hysteresis has an origin related to the intrinsic thermodynamics rather than to transport limitations. ${ }^{8}$

Another promising electrode candidate, alternative to carbon anodes in LIB, is Si because of its high specific theoretical capacity, $4200 \mathrm{~mA} \mathrm{~h} \mathrm{~g}^{-1}$ when is fully lithiated ( $\left.\mathrm{Li}_{22} \mathrm{Si}_{5}\right)$. Although, a considerable volumetric change and then a fast capacity fading is actually observed. For this reason, new materials are synthesized whose properties improve the cyclability, such as Si nanotubes (Si NT) which exhibit a reversible morphology, but it is still limited by low electron conductivity and ionic diffusivity. On the other hand, Si/Ge double-layered nanotube (Si/Ge DLNT) electrode shows improvements in structural stability and electrochemical kinetics. Ge shell favors the incorporation of Li-ions from the electrolyte to the semiconductor structure, since Ge has the higher electron conductivity that $\mathrm{Si}$ NT and allows to reduce the charge transfer resistance associated to the incorporation of Li-ion (Figure 9). ${ }^{26}$ The kinetic limitations are explained by impedance methods using the equivalent circuit presented in Figure 1e with additional $R C$ series subcircuit accounting for external SEI films.

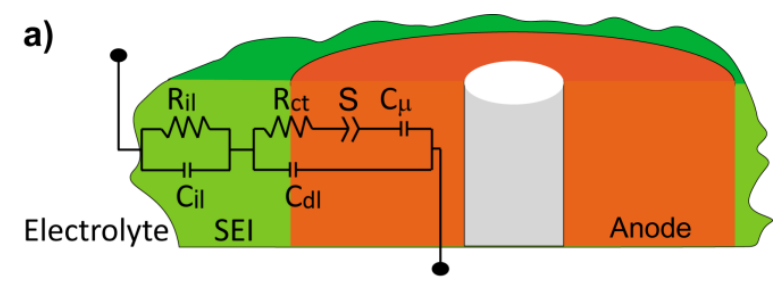

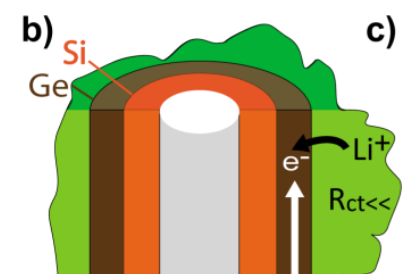

Si/Ge DLNT

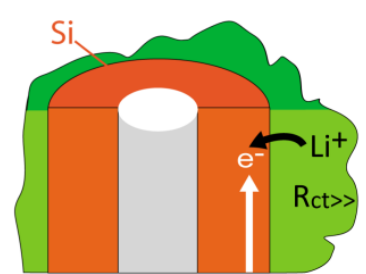

Si NT 
Figure 9 Schematic view of the relationship between equivalent circuit elements and electrode layer in $\mathrm{Si} / \mathrm{Ge}$ and Si NT electrodes. Reproduced (adapted) from ref. ${ }^{26}$ with permission from Royal Society of Chemistry.

The kinetic limitations are easily associated with a resistive process that occurs during the test in a quasi-steady state., the Si NT electrodes present clearly an arc at intermediate frequencies associated with mechanisms that occur in a double layer formed by the nanotubes. $R_{\mathrm{ct}}$ increases in the Si NT when the potential falls below $0.5 \mathrm{~V}$ vs. $\mathrm{Li} / \mathrm{Li}^{+}($ $R_{\mathrm{ct}} \approx 200 \Omega \mathrm{mg}$ ), while the incorporation of Ge layer reduces this parameter to values as low as $3 \Omega \mathrm{mg}$ (Figure 10). Again changes in Li-ion intake suffice to explain differences in charging kinetics.
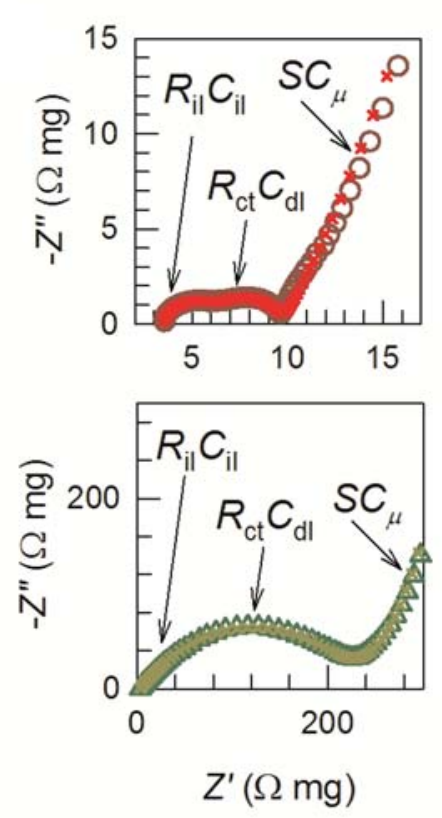

Figure 10 Impedance spectroscopy response of Si/Ge DLNT (circle) and Si NT (triangle) electrodes at $0.1 \mathrm{~V}$. Reproduced (adapted) from ref. ${ }^{26}$ with permission from Royal Society of Chemistry.

The conversion anode based on $\mathrm{ZnFe}_{2} \mathrm{O}_{4}$ exhibits a multi-step reaction process where intermediate phases of the $\mathrm{Li}-\mathrm{Zn}-\mathrm{Fe}-\mathrm{O}$ system are formed as precursors of amorphous $\mathrm{Li}_{2} \mathrm{O}$. The full reaction involves a complete reduction of the metal and an additional alloy with $\mathrm{Zn}: \mathrm{ZnFe}_{2} \mathrm{O}_{4}+9 \mathrm{Li}^{+}+9 \mathrm{e}^{-} \rightarrow \mathrm{LiZn}+2 \mathrm{Fe}^{0}+4 \mathrm{Li}_{2} \mathrm{O}$. A thorough study of the transition phases based on in-situ measurements XRD once the initial load-discharge galvanostatic confirm it. ${ }^{27}$ Figure 11 shows the main reactions taking place in lithiation-sliding zinc 
ferrite nanoparticles. Zinc ferrite electrodes have a high specific capacity and through a coating of carbon nanoparticles it is possible to maintain a fairly good kinetic performance in the high rate charge/discharge response. At lower charge states, cell voltage decreases abruptly, related to the formation of the interfaces, kinetically and energetically favored. At potentials below $0.5 \mathrm{~V}$ an increment in the specific capacity appears as a tail with respect to the conversion-related plateau. ${ }^{9}$

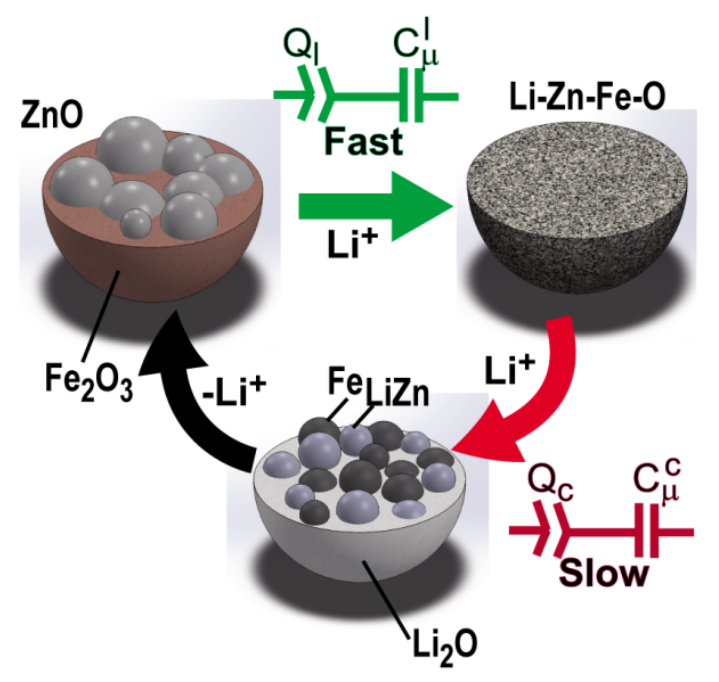

Figure 1 Schematic representation of the main process occurring in the carbon coated $\mathrm{ZnFe}_{2} \mathrm{O}_{4}-\mathrm{C}$ nanoparticle. Fast and slow charging subcircuits are drawn in connection to the involved reactions. Reproduced (adapted) from ref. ${ }^{9}$ with permission (2014) from American Chemical Society.

As commented upon previously, the electrodes involve a reorganization of the material of chemical and structural nature, it allows us to consider as limiting the conversion reaction itself. The whole equivalent circuit to be used for the electrode characterization is that depicted in Figure 1e. Here two reaction subcircuits are included accounting for the multistep lithiation mechanisms, which gives rise to two separate capacitive processes. The chemical capacitance exhibits then two contributions: $C_{\mu}^{\mathrm{c}}$ corresponding to the full conversion reaction at lower potentials, and $C_{\mu}^{1}$ produced by intermediate lithiation $\mathrm{Li}-\mathrm{Zn}-\mathrm{Fe}-\mathrm{O}$ phases (Figure 12). At lower potential, intermediate lithiation is masked by the huge $C_{\mu}^{\mathrm{c}}$ values exhibited by the conversion process. In both cases, a rather monotonic increment occurs toward lower potentials being $C_{\mu}^{\mathrm{c}}>C_{\mu}^{1}$ at a given charging state. It is interesting to compare here the capacitance extracted from EIS with the discharge curve derivative $-d Q / d V$ in Figure $12 \mathrm{a}$. 

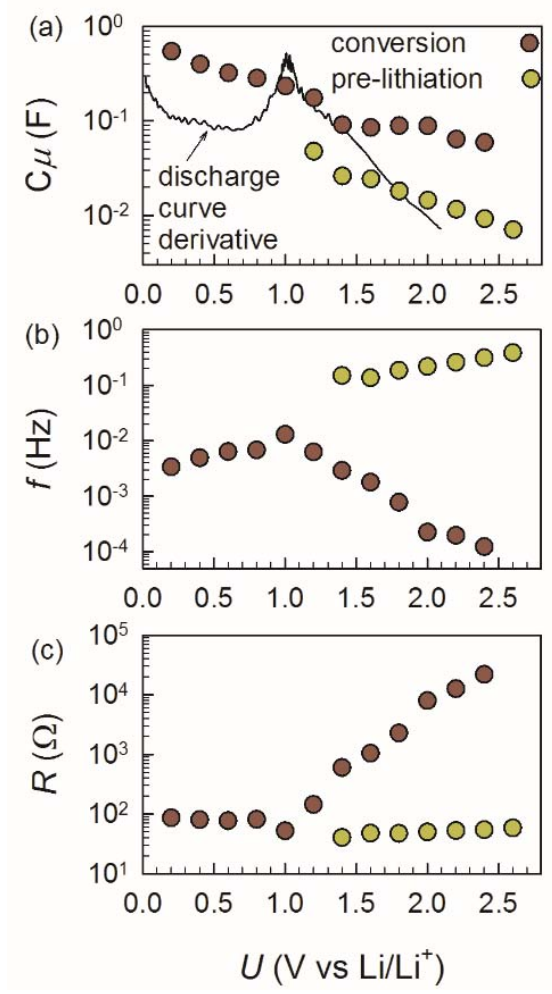

Figure 12 Fitting results using the equivalent circuit of Figure 1e showing (a) chemical capacitance $C_{\mu}$, (b) reaction frequency $f=\omega_{\mathrm{r}} / 2 \pi$, and (c) reaction equivalent resistance $R_{\mathrm{r}}$ for the prelithiation $\mathrm{Li}-\mathrm{Zn}-\mathrm{Fe}-\mathrm{O}$ phases formation, and the main conversion-alloying mechanism. In (a) solid line corresponds to the derivative of the discharge curve $-d Q / d V$ . Reproduced (adapted) from ref. ${ }^{9}$ with permission (2014) from American Chemical Society.

Figure 12a allows inferring that while the discharge curve derivative is reduced at potentials below the peak at $1.0 \mathrm{~V}$ (which corresponds to the voltage plateau), chemical capacitance exhibits higher values. This discrepancy can be understood by considering that discharge curve at $40 \mathrm{~mA} \mathrm{~g}^{-1}(\mathrm{C} / 20$ rate) is still far from equilibrium conditions where EIS has been measured. This would entail that the discharge curve plateau prolongs in the case of ultraslow rates giving as a consequence increasing capacitances toward lower potentials.

\section{$\mathrm{Li}_{-}-\mathrm{O}_{2}$ impedance response}

To enhance the energy density storage in LIB technology is required to advance beyond the $\mathrm{Li}^{+}$intercalation mechanisms. For $\mathrm{Li}-\mathrm{O}_{2}$ cathodes, the energy is stored by the direct 
reaction between $\mathrm{Li}^{+}$ions and $\mathrm{O}_{2}$ in a porous electrode that acts as an electric conductive substrate. This change in the chemistry of energy storage in the electrode is reflected in the EIS measurements which requires different equivalent circuit models that those reported in previous sections.

To elucidate the equivalent circuit model, EIS measurements were carried out in different cathodes during discharge process in presence (with) and absence (w/o) of $\mathrm{O}_{2}{ }^{28}$ Figure $13 \mathrm{a}$ shows two representations of these measurements: the impedance plot obtained at $2.6 \mathrm{~V}$ and the capacitance spectra $C=1 / i \omega Z$, versus the characteristic time (inverse of measuring frequency) in which the capacitive steps during the discharge process are represented by plateaus. In the absence of $\mathrm{O}_{2}$, the same impedance plot is registered in all the voltage range (between 4.2 and $2.2 \mathrm{~V}$ ), with an arc above $5 \mathrm{~Hz}$ related to a first plateau (blue square in Figure 13b), and a capacitive response below $5 \mathrm{~Hz}$ with the observation of a second plateau (orange square in Figure 13b). In the presence of $\mathrm{O}_{2}$, a similar response than that described for the absence of $\mathrm{O}_{2}$ is obtained at voltages above the $\mathrm{O}_{2}$ adsorption $\left(\mathrm{O}_{2}+\mathrm{e}^{-} \leftrightarrow \mathrm{O}_{2}^{-}, E^{0}=2.71 \mathrm{~V}\right)$. However, the impedance plot changes drastically at $2.6 \mathrm{~V}$, with the presence of a third arc between $5 \mathrm{~Hz}$ and $10 \mathrm{mHz}$, and followed by a diffusion tail. The capacity response (Figure 13b) also changes with the decrease of 1 order of magnitude of capacity in the second plateau followed by a steeply increase.

With this data, we proposed an equivalent circuit model (Figure 13c) in which the electrode discharge develops in three processes: (i) Interfacial phenomena ( $f>5 \mathrm{~Hz}$ ) that is $\mathrm{O}_{2}$ dependent. In the presence of $\mathrm{O}_{2}$ (Figure 13a), the impedance plot shows an extra arc ascribed to the solid film formed on the cathode containing $\mathrm{Li}_{2} \mathrm{O}_{2}$ and $\mathrm{Li}_{2} \mathrm{CO}_{3}$, products in the reaction between $\mathrm{Li}$ ions and $\mathrm{O}_{2}$. (ii) Electrical double layer capacitance, EDLC (10 $\mathrm{mH}<f<5 \mathrm{~Hz}$ ) that is $\mathrm{O}_{2}$ independent at voltages above $\mathrm{O}_{2}$ adsorption reaction. This process is related to the ion transport along the tortuous path of the porosity of the carbon matrix with concomitant surface accumulation. ${ }^{29}$ The decrease of almost one order of the extended $C_{\mathrm{dl}}$ indicates that the adsorption of $\mathrm{O}_{2}$ displaces the previous adsorbed $\mathrm{Li}^{+}$ions in a kinetic competition between $\mathrm{Li}^{+}$adsorption and consumption by oxygen reduction reaction (ORR). It provides information of the state of carbon surface. ${ }^{28}$ (iii) Chemical ORR capacitance $(f<10 \mathrm{mHz})$ that only appears in the presence of $\mathrm{O}_{2}$. The equivalent circuit model (Figure 13c) shows a new parallel branch to the extended $C_{\mathrm{dl}}$ subcircuit when the $\mathrm{O}_{2}$ starts to react (below $2.7 \mathrm{~V}$ ), containing the chemical capacitance, $C_{\mu}$, in series to the associated resistance, $R_{\mathrm{ORR}}$, accounting for the reduction reaction. Actually, 
more elements are needed related to the $\mathrm{O}_{2}$ diffusion through different layers, since the real part of the impedance (Z') increases in the tail below $10 \mathrm{mHz}$, but very slow measurements should be required for a more concise model.

a)

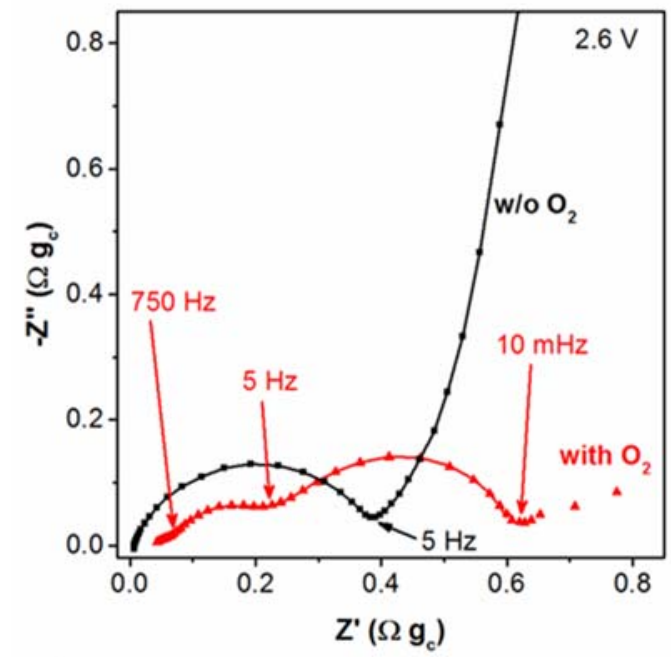

b)

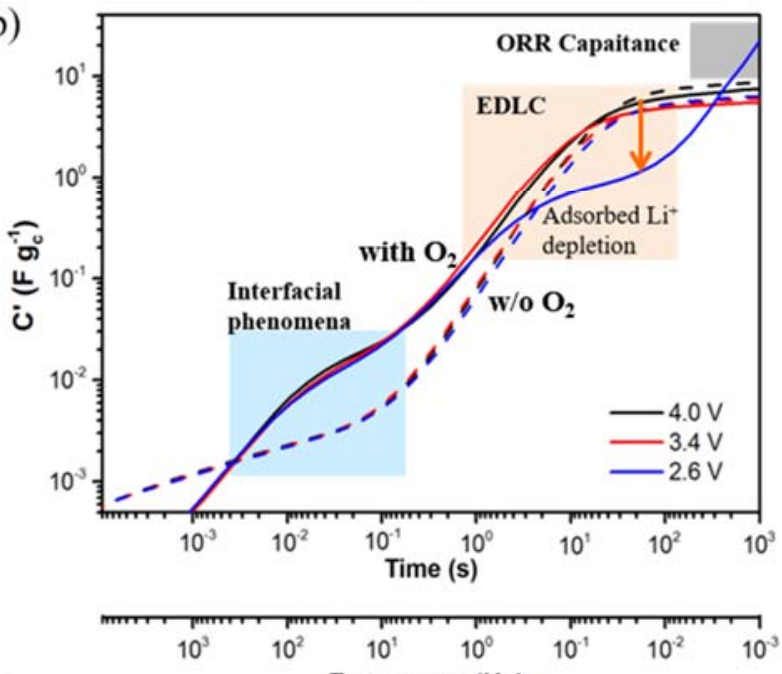

c)

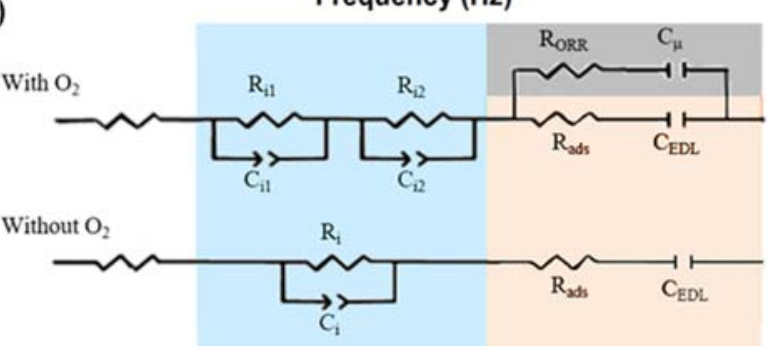

Figure 13 (a) Impedance plot at $2.6 \mathrm{~V} \mathrm{vs} \mathrm{Li}^{+} / \mathrm{Li}$, and (b) capacitance vs. characteristic time and frequency (in opposite order than usual, from fast to slow electrochemical processes for a more intuitive reading) for a carbon electrode in the presence (solid lines) and absence (dashed lines) of $\mathrm{O}_{2}$. (c) Equivalent circuit model for the system in the absence and presence of $\mathrm{O}_{2}$. Reproduced (adapted) from ref. ${ }^{28}$ with permission from WILEY-VCH. 
The main differences of our equivalent circuit model with others proposed in the literature for $\mathrm{Li}-\mathrm{O}_{2}$ batteries are two: the identification of the electrostatic capacitance related to the $\mathrm{Li}^{+}$adsorption, extended $C_{\mathrm{dl}}$, and the identification of the ORR related phenomena only at voltages and frequencies below $2.7 \mathrm{~V}$ and $10 \mathrm{mHz}$. In particular, three steps are also identified in the equivalent circuit models proposed previously, ${ }^{30,31}$ but assigned to different physical processes. In any way, the detailed analysis of the extended $C_{\mathrm{dl}}$ can be a useful tool to evaluate the mechanism of a cathode performance loss: clog of the porosity or coverage of the electric conductive substrate by the electrically insulating products $\left(\mathrm{Li}_{2} \mathrm{O}_{2}\right.$ and $\left.\mathrm{Li}_{2} \mathrm{CO}_{3}\right)$ as it has been reported in other studies. ${ }^{32}$

\section{Conclusions}

This Feature article presents a survey on the applicability of recently proposed equivalent circuit models to the analysis of the lithiation kinetics of several kinds of battery electrodes. These new models appear as a consequence of observing electrode mechanisms by means of standard electrochemical impedance spectroscopy. As explained previously, kinetic limitation to the charging process has been mainly understood in terms of hindrances to ionic or electronic transport. Here we introduce a novel concept that connects kinetic limitations to the electrode reduction itself. The low-frequency part of the impedance spectra conveys useful information about reaction limitations. For some oxides such as $\mathrm{LiFePO}_{4}, \mathrm{Li}\left[\mathrm{Ni}_{0.80} \mathrm{Co}_{0.06} \mathrm{Mn}_{0.14}\right] \mathrm{O}_{2}$, and $\mathrm{TiO}_{2}$ the newly proposed models has been proved to be useful for mechanism identification. The model is fully exploitable in the case of conversion and alloying electrodes for which a complete rearrangement of the material structure is expected. Here, several electrode materials with different morphologies $\left(\mathrm{FeOOH}, \mathrm{ZnFe}_{2} \mathrm{O}_{4}\right.$ and $\mathrm{Si} / \mathrm{Ge}$ ) have been summarized. In many cases, improvement in electrode rate capability correlates with the reduction in charge-transfer resistance favored by electronic contacting strategies. For the sake of completeness, the special instance of Li$\mathrm{O}_{2}$ electrodes are also included to present the great potential impedance analysis has on the analysis of electrical mechanisms, at interfaces as well as at particle bulk, in electrode materials.

\section{Conflicts of interest}

There are no conflicts to declare. 


\section{Acknowledgments}

Authors acknowledge fruitful discussions with Prof. Bisquert and Prof. Passerini. We thank financial support by Generalitat Valenciana under Prometeo Project (PROMETEO/2014/020), and Ministerio de Economía y Competitividad (MINECO) of Spain under Project (MAT2016-76892-C3-1-R). N.V. acknowledges Universitat Jaume I through FPI Fellowship Program (PREDOC/2015/54) and Project (UJI-B2016-35). SCIC of Universitat Jaume I is also acknowledged.

\section{References}

1. J. Bisquert, Nanostructured Energy Devices: Equilibrium Concepts and Kinetics, CRC Press, Boca Raton, FL, 2015.

2. J. Jamnik and J. Maier, Phys. Chem. Chem. Phys., 2001, 3, 1668-1678.

3. J. Bisquert, Phys. Chem. Chem. Phys., 2003, 5, 5360-5364.

4. J. Bisquert and A. Compte, J. Electroanal. Chem., 2001, 499, 112-120.

5. G. Garcia-Belmonte, Z. Pomerantz, J. Bisquert, J.-P. Lellouche and A. Zaban, Electrochim. Acta, 2004, 49, 3413-3417.

6. J. García-Cañadas, F. Fabregat-Santiago, I. Porqueras, C. Person, J. Bisquert and G. Garcia-Belmonte, Solid State Ionics, 2004, 175, 521-525.

7. J. Bisquert, G. Garcia-Belmonte and A. Pitarch, ChemPhysChem, 2003, 4, 287-292.

8. C. Xu, Y. Zeng, X. Rui, J. Zhu, H. Tan, A. Guerrero, J. Toribio, J. Bisquert, G. GarciaBelmonte and Q. Yan, J. Phys. Chem. C, 2013, 117, 17462-17469.

9. F. Martinez-Julian, A. Guerrero, M. Haro, J. Bisquert, D. Bresser, E. Paillard, S. Passerini and G. Garcia-Belmonte, J. Phys. Chem. C, 2014, 118, 6069-6076.

10. M. D. Levi and D. Aurbach, J. Phys. Chem. B, 2004, 108, 11693-11703.

11. J. P. Meyers, M. Doyle, R. M. Darling and J. Newman, J. Electrochem. Soc., 2000, 147, 2930-2940.

12. J. Bisquert, G. Garcia-Belmonte, F. Fabregat-Santiago and A. Compte, Electrochem. Commun., 1999, 1, 429-435.

13. K.-J. Park, B.-B. Lim, M.-H. Choi, H.-G. Jung, Y.-K. Sun, M. Haro, N. Vicente, J. Bisquert and G. Garcia-Belmonte, J. Mater. Chem. A, 2015, 3, 22183-22190.

14. N. Vicente, M. Haro, D. Cíntora-Juárez, C. Pérez-Vicente, J. L. Tirado, S. Ahmad and G. Garcia-Belmonte, Electrochim. Acta, 2015, 163, 323-329.

15. G.-A. Nazri and G. Pistoia, eds., Lithium Batteries: Science and Technology, Springer Science+Business Media, New York, 2009.

16. S. Martha, E. Markevich, V. Burgel, G. Salitra, E. Zinigrad, B. Markovsky, H. Sclar, Z. Pramovich, O. Heik and D. Aurbach, J. Power Sources, 2009, 189, 288-296.

17. H.-J. Noh, S. Youn, C. S. Yoon and Y.-K. Sun, J. Power Sources, 2013, 233, 121-130.

18. B. Hwang, Y. Tsai, D. Carlier and G. Ceder, Chem. Mater., 2003, 15, 3676-3682.

19. A. K. Padhi, K. Nanjundaswamy and J. B. Goodenough, J. Electrochem. Soc., 1997, 144, 1188-1194.

20. G. Li and P. G. Pickup, Phys. Chem. Chem. Phys., 2000, 2, 1255-1260.

21. P. Acevedo-Peña, M. Haro, M. E. Rincón, J. Bisquert and G. Garcia-Belmonte, J. Power Sources, 2014, 268, 397-403.

22. R. Hass, J. García-Cañadas and G. Garcia-Belmonte, J. Electroanal. Chem., 2005, 577, 99105.

23. N. Vicente and G. Garcia-Belmonte, J. Phys. Chem. Lett., 2017, 8, 1371-1374.

24. N. Vicente and G. Garcia-Belmonte, Adv. Energy Mater., 2017, 7, 1700710.

25. J. Cabana, L. Monconduit, D. Larcher and M. R. Palacin, Adv. Mater., 2010, 22, E170E192. 
26. M. Haro, T. Song, A. Guerrero, L. Bertoluzzi, J. Bisquert, U. Paik and G. GarciaBelmonte, Phys. Chem. Chem. Phys., 2014, 16, 17930.

27. D. Bresser, E. Paillard, R. Kloepsch, S. Krueger, M. Fiedler, R. Schmitz, D. Baither, M. Winter and S. Passerini, Adv. Energy Mater., 2013, 3, 513-523.

28. M. Haro, N. Vicente and G. Garcia-Belmonte, Adv. Mater. Interfaces, 2015, 2, 1500369.

29. G. Rasines, P. Lavela, C. Macías, M. Haro, C. Ania and J. Tirado, J. Electroanal. Chem., 2012, 671, 92-98.

30. J. Højberg, B. D. McCloskey, J. Hjelm, T. Vegge, K. Johansen, P. Norby and A. C. Luntz, ACS Appl. Mater. Interfaces, 2015, 7, 4039-4047.

31. I. Landa-Medrano, I. R. de Larramendi, N. Ortiz-Vitoriano, R. Pinedo, J. I. R. de Larramendi and T. Rojo, J. Power Sources, 2014, 249, 110-117.

32. B. D. McCloskey, A. Speidel, R. Scheffler, D. C. Miller, V. Viswanathan, J. S. Hummelshøj, J. K. Nørskov and A. C. Luntz, J. Phys. Chem. Lett., 2012, 3, 997-1001. 